\title{
Indices, Graphs and Null Models: Analyzing Bipartite Ecological Networks
}

\author{
Carsten F. Dormann ${ }^{*}, 1$, Jochen Fründ ${ }^{2}$, Nico Blüthgen ${ }^{3}$ and Bernd Gruber ${ }^{1}$ \\ ${ }^{1}$ Helmholtz Centre for Environmental Research, Department Computational Landscape Ecology, Permoserstr. 15, \\ 04318 Leipzig, Germany \\ ${ }^{2}$ Agroecology, University of Göttingen, Waldweg 26, 37073 Göttingen, Germany \\ ${ }^{3}$ Department of Animal Ecology and Tropical Biology, University of Würzburg, Biozentrum, Am Hubland, 97074 \\ Würzburg, Germany
}

\begin{abstract}
Many analyses of ecological networks in recent years have introduced new indices to describe network properties. As a consequence, tens of indices are available to address similar questions, differing in specific detail, sensitivity in detecting the property in question, and robustness with respect to network size and sampling intensity. Furthermore, some indices merely reflect the number of species participating in a network, but not their interrelationship, requiring a null model approach. Here we introduce a new, free software calculating a large spectrum of network indices, visualizing bipartite networks and generating null models. We use this tool to explore the sensitivity of 26 network indices to network dimensions, sampling intensity and singleton observations. Based on observed data, we investigate the interrelationship of these indices, and show that they are highly correlated, and heavily influenced by network dimensions and connectance. Finally, we re-evaluate five common hypotheses about network properties, comparing 19 pollination networks with three differently complex null models: 1 . The number of links per species ("degree") follow (truncated) power law distributions. 2. Generalist pollinators interact with specialist plants, and vice versa (dependence asymmetry). 3. Ecological networks are nested. 4. Pollinators display complementarity, owing to specialization within the network. 5. Plant-pollinator networks are more robust to extinction than random networks. Our results indicate that while some hypotheses hold up against our null models, others are to a large extent understandable on the basis of network size, rather than ecological interrelationships. In particular, null model pattern of dependence asymmetry and robustness to extinction are opposite to what current network paradigms suggest. Our analysis, and the tools we provide, enables ecologists to readily contrast their findings with null model expectations for many different questions, thus separating statistical inevitability from ecological process.
\end{abstract}

Keywords: Connectance, linkage density, nestedness, network, pollination web, specialization.

\section{INTRODUCTION}

The long-standing quest of community ecology is to find patterns in species assemblages (Strong et al. 1984; Diamond and Case 1986). A first step is to understand why species are as abundant as they are, why they occur where they occur, and how species richness emerges from evolution and environmental constraints (Krebs 2002). A second step is to find generalizable pattern beyond these first-order processes: how communities assemble (Diamond 1975; Weiher and Keddy 1999), which species traits make communities stable (May 1973; McCann 2000), and which processes govern community structure (Berlow 1999; Berlow et al. 2004).

In recent years, bipartite interaction networks (in ecology mainly interaction networks with two trophic levels) gained

\footnotetext{
*Address correspondence to this author at the Helmholtz Centre for Environmental Research, Department Computational Landscape Ecology, Permoserstr. 15, 04318 Leipzig, Germany; Tel: ++49 341235 1946; Fax: ++49 341235 1939; E-mail: carsten.dormann@ufz.de

Handling Editor: Judi Hewitt
}

more prominence. This research has produced new indices for interaction networks (e.g. Bersier et al. 2002; Blüthgen et al. 2006), detected various patterns in them (e.g. Dunne et al. 2002; Olesen and Jordano 2002; Jordano et al. 2003; Vázquez and Aizen 2004) and provided new stimulus to research into evolutionary mechanisms within communities (e.g. Lewinsohn et al. 2006; Rezende et al. 2007; Mouillot et al. 2008) and to the link between land use/landscape structure, network structure and ecosystem function (Vázquez and Simberloff 2003; Fortuna and Bascompte 2006; Tylianakis 2008).

While many of the proposed indices may provide insight into ecologically or evolutionary important aspects of bipartite networks, most of them have not been scrutinized for their sensitivity to first-order aspects of the network analyzed, such as its dimensions (number of species in higher or lower trophic level), asymmetry of network dimensions (Blüthgen et al. 2007), marginal abundance distributions (Vázquez and Aizen 2003; Blüthgen et al. 2008) or number of observations per species (interaction density: Nielsen and Bascompte 2007). There is a real risk 
that some indices heralded as ecologically significant descriptors of networks may actually be mere reflections of the sampling properties of the underlying network (Blüthgen et al. 2008). If so, the ecological interest of network would lie in the question of why a network has a given number of species, and why they are as abundant as they are, but not in the way they interact, since that would have to be considered as random. To correct for such artifacts, null models should and can be employed (Gotelli and Graves 1996; Vázquez and Aizen 2003, 2004; Vázquez et al. 2007). Finally, there are very few studies that assess several different network indices
(Blüthgen et al. 2007; Nielsen and Bascompte 2007; Vázquez et al. 2007; Blüthgen et al. 2008), hence it remains unclear how correlated their response are and thus whether they respond to the same network properties.

In this study, we provide A) a brief overview of common indices and metrics of bipartite interaction networks and B) a systematic evaluation of how first-order network features and scarce observations affect them. Specifically, we investigate how network indices are affected by network dimensions, sampling intensity, singleton observations and
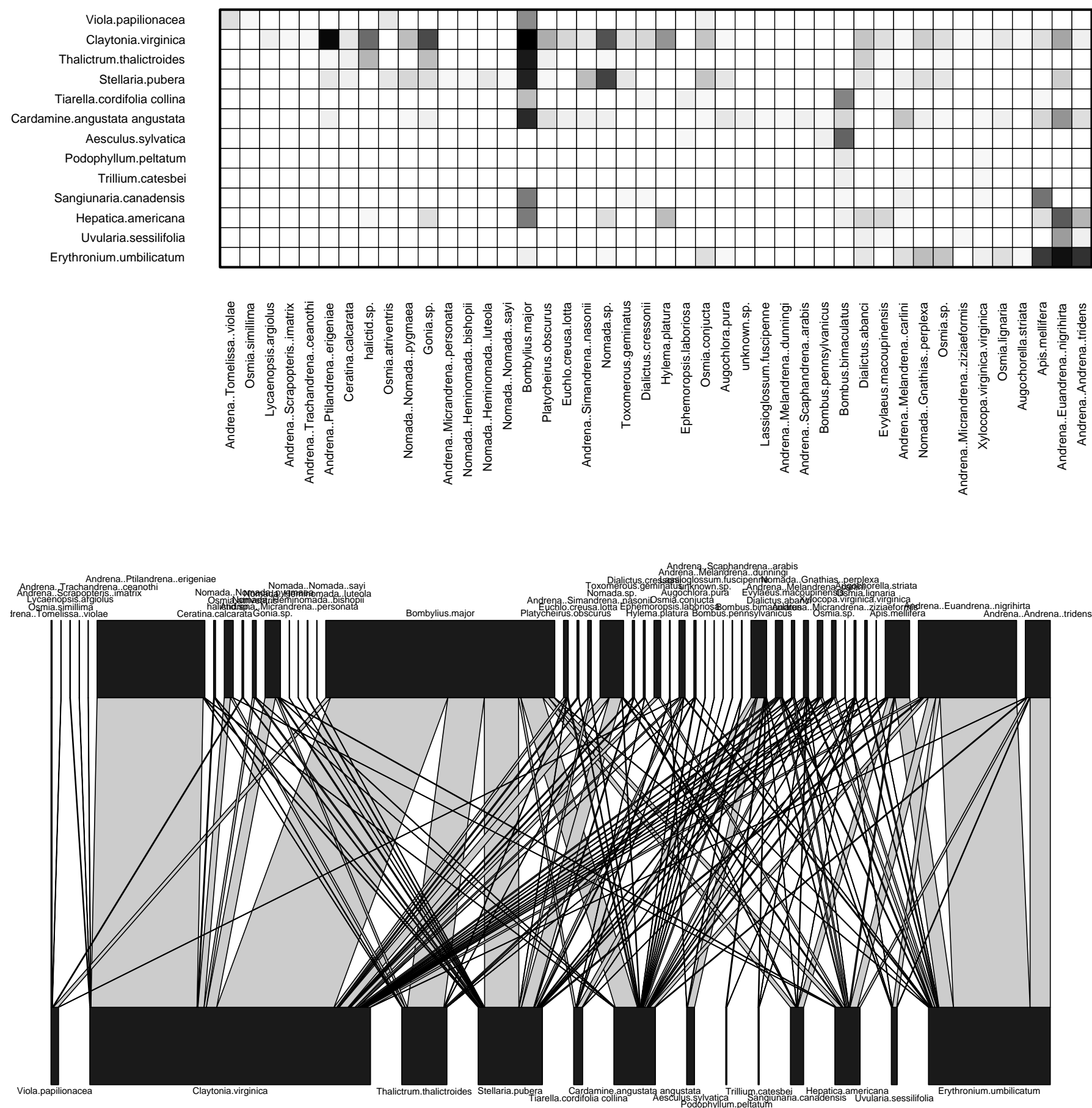

Fig. (1). A network matrix (top) and its bipartite graph (bottom). Shading of matrix entries indicates number of observations. Sequence of species is identical in both network visualizations (minimized crossing of lines). Dataset: Motten (1982). 
errors. This leads to C) an analysis of the interrelationship of bipartite network indices. Finally, D), we re-assess some of the most prominent hypotheses on network topology with data from 19 pollination networks by comparing observed pattern to expectations based on different null models. To allow for maximum reproducibility, standardization and transparency, we also provide a new software tool for the visualization and analysis of bipartite networks.

\section{BIPARTITE NETWORK INDICES}

For simplicity, we refer to all pattern-reporting statistics, metrics, indices and statistics as indices. We compiled a list of indices from the literature on interaction networks, which is unlikely to be exhaustive, nor will every index describe an ecological process or hypothesis. Furthermore, not all indices used in the exploration of multitrophic food webs have an ecological meaning for bipartite networks. On the other hand, some indices invented for biogeographical analyses can be employed for bipartite networks, since they describe pattern caused by (lack of) competition within a trophic level.

We define the following entities:

$L \quad$ number of realized links in a network

I number of lower trophic level species (e.g. plants, prey)

$J$ number of higher trophic level species (e.g. pollinators, parasitoids)

$m \quad$ total number of interactions for all species

$a_{i j} \quad$ number of interactions between species $i$ from the lower and species $j$ from the higher trophic level, also referred as "link weight"

$A_{i} \quad$ total number of interactions of species $i$ from the lower trophic level, thus $A_{i}=\sum_{j=1}^{J} a_{i j}$

$A_{j} \quad$ total number of interactions of species $j$ from the higher trophic level, thus, $A_{j}=\sum_{i=1}^{I} a_{i j}$

We refer to a "link" as one or several interactions between a pair of species (one from the lower, one from the higher trophic level), and to the number of observations of a link as "interactions". For example, in Fig. (1a) the number of non-white cells equals $L$, while the width of the black bars in Fig. (1b) represents the number of observed interactions. On average, the number of interactions per species pair is $\overline{a_{i j}}=m /(I J)$ and referred to as "observation density".

The network indices investigated here are defined as follows:

\section{(a) Indices Based on Unweighted Links (Qualitative Webs)}

\section{Links per Species}

Mean number of links per species, $\bar{L}_{x}=L /(I+J)$.

\section{Connectance}

Realized proportion of possible links. $C=L /(I J)$. This is the standardized number of species combinations often used in co-occurrence analyses (Gotelli and Graves 1996); also identical to 2-species motifs sensu Milo et al. (2002).

\section{Cluster Coefficient}

Mean, across all species, of the number of realized links divided by the number of possible links for each species (i.e. average per-species connectance). The cluster coefficient was introduced by Watts and Strogatz (1998) as one of two criteria defining a small-world network.

\section{Web Asymmetry}

Balance between numbers in the two levels $W=(I-$ $J) /(I+J)$. Positive numbers indicate more lower-trophic level species, negative more higher-trophic level species; rescaled to $[-1,1]$ (see Blüthgen et al. 2007).

\section{Number of Compartments}

Compartments are sub-sets of the web which are not connected (through either higher or lower trophic level) to another compartment (Jordan blocks in the mathematical nomenclature). Proposed by Pimm (1982) or, in a less strict version, by Dicks, Corbet and Pywell (2002), and argued to reveal evolutionary processes (Guimarães et al. 2007a). If several compartments exist, we can also compute compartment diversity as a measure of their size heterogeneity (Tylianakis et al. 2007).

\section{Extinction Slopes}

Exponent ("slope" $a$ ) of a power function $\left(y=1-x^{a}\right)$ fitted to the secondary extinction sequence in one trophic level, following random extermination of species in the other trophic level (Memmott et al. 2004). The higher the slope estimate, the less the network is affected by extinctions.

\section{Degree Distribution}

The number of links of a species is also called the species' degree. Jordano et al. (2003) proposed that plantanimal networks may show scale invariance, as indicated by the presence of a power law in species degrees. They report on consistently better fits of the truncated power law, hypothesizing that such patterns may arise from morphological mismatch or phenological uncoupling. The crucial step for this analysis is how often an exponential, power-law or truncated power-law provides the best fit to the data. We calculate degree distributions for the species of each trophic level separately.

\section{Mean Number of Shared Hosts}

The simplest possible measure of co-occurrence and hence similarity in host preferences (Roberts and Stone 1990; Stone and Roberts 1992).

\section{Togetherness}

Mean number of co-occupancies across all species-hostcombinations; the whole matrix is scanned for submatrices of the form $\left(\begin{array}{ll}0 & 1 \\ 0 & 1\end{array}\right)$, representing perfect matches of co- 
presences and co-absences. These are counted for each pairwise species combination, and averaged (Stone and Roberts 1992).

\section{C-Score}

Mean (normalized) number of checkerboard combinations across all higher trophic level species. Values close to 1 indicate that there is evidence for disaggregation, e.g. through competition. Value close to 0 indicate aggregation of species (i.e. no repelling forces between species) (Stone and Roberts 1992). In contrast, low co-occurrence values indicate competitive displacement among species (Diamond 1975).

\section{V-Ratio}

Variance-ratio of species numbers to individual numbers within species. Values larger than 1 indicate positive aggregation, values between 0 and 1 indicate disaggregation of species (Schluter 1984).

\section{Nestedness}

A measure of departure from systematic arrangement of species by niche width. The nestedness 'temperature' $T\left(0^{\circ}-\right.$ 100': Atmar and Patterson 1993), measures the departure from a perfectly nested interaction matrix. $T=0^{\circ}$ is defined for maximum nestedness: when rows and columns are ordered by decreasing number of links, links of each row and column exactly match the previous ones or represent a subset of them. Originally proposed for species on islands of different size and distance from mainland (Patterson and Atmar 1986; Atmar and Patterson 1993; Hausdorf and Hennig 2007; Moore and Swihart. 2007), nestedness was recently introduced into the analysis of bipartite networks (Bascompte et al. 2003; Guimarães et al. 2006; Lewinsohn et al. 2006; Guimarães et al. 2007b). However, recently the concept of nestedness and its quantification has received criticism and extensions (Brualdi and Sanderson 1999; Almeida-Neto et al. 2007; Moore and Swihart 2007; Almeida-Neto et al. 2008). For example, Blüthgen et al. (2008) showed that nested patterns are expected by random associations between species and that null models underlying $\mathrm{H}_{2}$ ' represent quantitatively nested networks.

\section{(b) Indices Based on Weighted Links (Quantitative Webs)}

\section{Generality}

Mean number of prey species per predator. If links are unweighted, generality is $G=L / J$. For weighted links, Bersier et al. (2002) proposed to use the 'effective' mean number of links as arithmetic mean $G_{q}=\frac{1}{J} \sum_{j=1}^{J} 2^{H_{j}}$ or as weighted mean $G_{q w}=\sum_{j=1}^{J} \frac{A_{j}}{m} 2^{H_{j}} \cdot H_{\mathrm{j}}$ is the Shannon diversity of interactions for predator species $j$, i.e. $H_{j}=-\sum_{i=1}^{I}\left(\frac{a_{i j}}{A_{j}} \cdot \ln \frac{a_{i j}}{A_{j}}\right)$. In the bipartite package, $G_{\mathrm{qw}}$ is implemented.

\section{Vulnerability}

Mean number of predator per prey. Analogous to generality, Bersier et al. (2002) proposed the effective mean number of links (replace $j$ by $i$ and $J$ by $I$ in the equation for $\left.G_{q w}\right)$.

\section{Weighted Linkage Density}

Mean number of links per species (see above), but weighted by the number of interactions. Bipartite uses the weighted mean across the species, following Bersier et al. (2002). Since generality and vulnerability are known, weighted linkage density is given as their arithmetic mean (Tylianakis et al. 2007): $L_{q}=0.5\left(\sum_{j=1}^{J} \frac{A_{j}}{m} 2^{H_{j}}+\sum_{i=1}^{I} \frac{A_{i}}{m} 2^{H i}\right)$

\section{Interaction Evenness}

Shannon's evenness of network interactions: $E_{S}=\frac{-\sum_{i} \sum_{j} p_{i j} \ln p_{i j}}{\ln L}$. It may be preferable to divide by $\ln I J$, arguing that non-existing links are also contributing information. Several other measures of evenness can be considered. One, Alatalo interaction evenness (Alatalo 1981), was proposed by Müller et al. (1999b) as particularly suitable for networks. Let $p_{i j}=a_{i j} / m$ represent the proportion of interactions in a cell, $N_{1}=\exp \left(-\sum_{i} \sum_{j} p_{i j} \ln p_{i j}\right)$ the antilogarithmic Shannon entropy, and $N_{2}=\left(\sum_{i} \sum_{j} p_{i j}^{2}\right)^{-1}$ the reciprocal of Simpson's index. Then Alatalo's evenness is defined as: $F_{2.1}=\left(N_{2}-1\right) /\left(N_{1}-1\right)$ (see Alatalo 1981, p. 200, for origin of this index).

$\boldsymbol{H}_{\mathbf{2}}$ ': A network-level measure of specialization, based on the deviation of a species' realized number of interactions and that expected from each species' total number of interactions (Blüthgen et al. 2006). The underlying equation is the same as interaction diversity (Shannon $\mathrm{H}_{2}$ ), but the value computed for the given network $\left(\mathrm{H}_{2}\right)$ is standardized against the minimum $\left(H_{2 \min }\right)$ and maximum $\left(H_{2 \min }\right)$ possible for the same distribution of interaction totals: $H_{2}{ }^{\prime}=\frac{H_{2 \max }-H_{2}}{H_{2 \max }-H_{2 \min }}$. The resulting $H_{2}$ ' ranges between 0 (no specialization) and 1 (perfect specialisation for given interaction totals).

\section{Interaction Strength Asymmetry}

Quantifies the imbalance between the interaction strengths of a species pair. Bascompte et al. (2006) argue that specialised pollinators will be matched up with generalist plants, and vice versa. Interaction strength (or 'dependence' sensu Jordano (1987) and Bascompte et al. (2006)) for species $i$ in terms of its partner species $j$ can be defined by the proportion of their interactions $a_{i j}$ of the total interactions recorded for $i\left(A_{i}\right)$, thus $b_{i j}=a_{i j} / A_{i}$. The reciprocal interaction strength for species $j$ to its partner $i$ is then $b_{j i}=a_{i j} / A_{j}$. Asymmetries of interaction strength can 
be defined as $A S_{i j}=\left(b_{i j}-b_{j i}\right) /\left(b_{i j}+b_{j i}\right)$ (see Bascompte et al. 2006 for a similar definition of 'dependence' asymmetry where the absolute values of $A S_{i j}$ are calculated). The interaction strength asymmetry of a network is the grand mean of $A S_{i j}$. One problem with this index is that singleton species receive very high influence, indicating high dependence, while in fact they only represent rare species. In our version of interaction strength asymmetry, all singleton species are omitted from the network. Blüthgen et al. (2007) showed that mean dependence asymmetry in a network can be almost entirely explained by web asymmetry (see above) and proposed a modification based on their species-level $d_{\mathrm{i}}$ 'index which they call specialization asymmetry. We analyze both a variation on Bascompte et al.'s dependence asymmetry (where we allow values to be positive or negative) and specialization asymmetry (based on logtransformed specialization values $d_{\mathrm{i}}$ ' for each species). In either case positive values indicate a higher specialization of the higher trophic level.

\section{Niche Overlap}

Mean similarity in interaction pattern between species of the same trophic level, calculated e.g. as Horn-Morisita similarity (Krebs 1989) or as Bray-Curtis similarity (Mouillot et al. 2008). Values near 0 indicate no common use of niches, 1 indicates perfect niche overlap.

We have tried to incorporate as many of commonly used indices as possible, but this list will not be exhaustive. One index we had to exclude because we do not, yet, have an algorithm implementing it, is modularity (Watts and Strogatz 1998; Olesen et al. 2007).

\section{METHODS}

\section{The Bipartite Package}

We provide software to calculate all of the above indices, visualize networks and create several different null models. This software comes in the form of the R-package bipartite and is available freely and open source (Dormann et al. 2008). R itself is available at www.r-project.org ( $R$ Development Core Team 2008). bipartite comprises functions to calculate all network indices presented here (function networklevel) as well as several other calculated at the level of the individual species (function specieslevel, not presented in this study), along with two ways to visualize bipartite networks (functions visweb and plotweb: Fig. 1), two null models (functions shuffle.web and swap.web, the Patefield algorithm presented below is already implemented in another package) and a simple generic function to construct artificial networks with approximately lognormally distributed marginal abundances (function genweb). We included 19 pollination networks as datasets in the bipartite package, both as resource and to illustrate the functionality of bipartite's functions, taken from the interaction web database of the National Center for Ecological Analysis and Synthesis (NCEAS), which are introduced below. Most importantly, all functions are documented in detail and the source code can be inspected for implementation details. Since bipartite is an open source product users can modify and add functionality and thus adapt the package to meet their own requirements. Please contact the authors for any further questions.

For some indices presented here, other software is available. In particular, nestedness can be calculated by the Nestedness Temperature Calculator (Atmar and Patterson 1993), Aninhado (Guimarães and Guimarães 2006) and Binmatnest (Rodríguez-Gironés and Santamaría (2006), which is the program underlying the nestedness calculations presented here and also implemented in the R-package vegan (Oksanen et al. 2008). Ecosim (Gotelli and Entsminger 2001) allows the calculation of biogeographic indices (such as $C$-score and $V$-ratio), while Pajek (Batagelj and Mrvar 2003) is a software for food web analysis and visualization. Degree distributions can also be fit using the degreenet package (Handcock 2003) of the statnet bundle for $\mathrm{R}$ (Handcock et al. 2003), which specifically addresses exponential family random graph models.

\section{Simulations and Analyses}

We carried out a systematic simulation study on random networks to compare the above-listed indices and to evaluate the effect of several network properties on the indices. Specifically, we were interested in two properties that differ widely between published pollination networks, network dimensions and sampling intensity, and the effect of singleton observations on the index estimates (since Kossinets 2006 has shown missing data to cause instability of network indices). In addition, we analyzed 19 published pollination networks for all the above indices and compared their values with those from two different null models.

\section{Generating Synthetic Networks}

Our simulations are based on observed values, as found in the pollination networks published in the NCEAS interaction webs database (http://www.nceas.ucsb.edu/ interactionweb and included in the R-package bipartite). At the time of this study, 19 different quantitative pollination networks were available. Since our data are based on pollination networks, we refer to the higher and lower trophic level as pollinators and plants, respectively, although the results are independent of the study system and hence also apply to non-mutualistic networks (such as hostparasitoid systems).

Each network is characterized by four values: number of plants, number of pollinators (the two dimensions of the network), sampling intensity (mean number of interactions per possible links in the network) and the standard deviation of the marginal distributions. To generate a network, we assumed log-normal distribution of plant and pollinators with a sampling intensity of $m=2$ (median of NCEAS' pollination webs) and a standard deviation of $s=1.5$ (the median of both rows and columns of NCEAS' pollination webs). With $I \cdot J$ representing the total number of possible interactions in a network, the mean of each trophic level's lognormal marginal distribution is $\mu_{I}=\log (\mathrm{J} \cdot \mathrm{m})-0.5 \cdot \mathrm{s}^{2}$ and $\mu_{J}=\log (I \cdot m)-0.5 \cdot s^{2}$ for plants and pollinators, respectively. We drew random numbers from the thus specified marginal distributions, observing that each of the marginal sums had to add to exactly $L$ (which may lead to deviations from a lognormal distribution for low sampling intensities), and 
used them as input for a Patefield algorithm for constructing random 2-way tables with given marginal totals (Patefield 1981, implemented in $\mathrm{R}$ as function r2dtable and also the basis for most re-sampling-based contingency table tests such as $\mathrm{X}^{2}$ or Fisher test).

For the analysis of network dimensional effects, we varied $I$ and $J$ independently from 4 to 238, yielding 110 different network dimensions. $I$ and $J$ were chosen in such a way that network asymmetry was independent of network size but systematically explored the full range of possible asymmetries (see Fig. 2, second row, fifth panel). For the analysis of effects of sampling intensity, we varied $m$ in 9 levels in the range observed in the NCEAS pollination networks: $\mathrm{SI}=0.1,0.2,0.3,0.5,1,2,3,5$ and $10 . I$ and $J$ were set to 10 and 30, respectively, representing the median sizes of NCEAS pollination networks. For both analyses, 20 replicates were generated.

All randomly generated webs were stored and pooled for the third analysis, that of singleton observation removal effects. Here we deleted all links recorded with only one interaction and then removed all species with no remaining link from the network.

\section{Real Pollination Networks and Null Models}

We used all 19 pollination networks with quantitative data from the NCEAS database (see http://www.nceas.ucsb. edu/interactionweb for details). To investigate how much of the observed pattern in the different indices were due to network dimensions, sampling intensity, marginal distributions and realized number of links, we ran three null models (Gotelli and Graves 1996; for other examples of null models in network analysis see, e.g., Burns 2007, Ulrich and Gotelli 2007, Vázquez et al. 2003 and Guimarães et al. 2007a). As long as only phenomenological models for generating food web structure are available (Allesina et al. 2008; Clauset et al. 2008; Williams and Martinez 2008), null models are a valuable aid in testing the success of pattern generating processes.

Null model I is the same as for the artificial networks, i.e. the Patefield algorithm, based on the observed network marginal totals (Blüthgen et al. 2008 showed that different modifications of this approach yield near-identical findings). This null model is also called $\mathrm{R}_{\mathrm{ff}}$ (see Moore and Swihart 2007, for a detailed discussion). Null model II is a shuffling algorithm, which scatters the observed interaction values in the matrix while maintaining the network dimensions. Technically this is achieved by first filling the network matrix diagonal and then randomly allocating the remaining interactions. Null model III is a swapping algorithm employed on a network generated by null model I, but it additionally constraints connectance to the same value as found in the original web. Technically, it starts with a Patefield-generated matrix and then randomly selects $2 \times 2$ submatrices without zeros and subtracts the minimum values of the diagonal from the diagonal (thereby generating an empty cell) and adds this value to the values on the minor diagonal. This maintains marginal totals while reducing the number of links (see Gotelli 2000, whose binary version of this swapping algorithm inspired our null model III). This procedure is repeated until the number of links in the null model is equal to that of the observed network. Our null model III should yield similar results to the null model of Vázquez and Aizen (2003), although it is based on a different principle.

Null models will always be contentious (Gotelli and Graves 1996), because the level of detail they contain and the degree of constraint they impose is somewhat arbitrary. Our three null models are not comprehensive, but they allow us to elucidate which role marginal abundance distributions and realized number of links play for the various network indices presented here. These three null models are designed to be complementary, because they constrain the random network generation in different ways. The comparison that is most important to grasp is that between null model I (Patefield) and III (swap). The Patefield algorithm creates null models with marginal totals identical to those of the observed web, i.e. some species are common, other rare. In most cases, however, these null model matrices are less sparse than the observed, i.e. they contain more links. Null model III additionally constraints this property: here, the number of links in the null model is equal to those of the observed network, as are the marginal totals. Constraining the number of links is analogous to some characteristic ecological and/or evolutionary processes, variously described as "trait mismatch", "morphological barriers" or "forbidden links" (Jordano et al. 2006; Stang et al. 2006; Santamaría and Rodríguez-Gironés 2007; Stang et al. 2007), and attributed to co-evolution of flowers and pollinators or hosts and parasitoids, respectively (but see Vázquez et al. 2005). Thus, if null model I does not reproduce the observed data while null model III does, this may be interpreted as a real ecological property of the network, not a mere consequence of the fact that some species are more common than others.

The shuffle-algorithm (null model II) also keeps the number of links constant, but yields marginal distributions very different from the original. Comparing null model II to null model III allows us to elucidate if the number of links is more important than the marginal distributions. That means if both, null model II and III, reproduce the observed pattern, then it must be attributed primarily to the degree of realized links in the network, not to the shape of species abundance distributions. The null networks from algorithm "shuffle" are often highly "unrealistic".

Finally, we investigated the effect of perturbing the observed network for the network indices, simulating for example misidentification, typos or aberrant behavior of pollinators. To do so, we randomly selected one of the observed interactions and added it to a random cell of the interaction matrix (allowing re-assignment to the cell of origin). The chance of assigning it to an unobserved, new pollinator species was set to $1 /(J+1)$. This procedure was repeated until 5,10 or $20 \%$ of all interactions were rearranged, thus simulating moderate to strong errors in the data.

Null models and perturbations were replicated 1000 times, which took a desktop computer approximately four weeks (four days for generating the networks and three and a half weeks for computing the indices). 


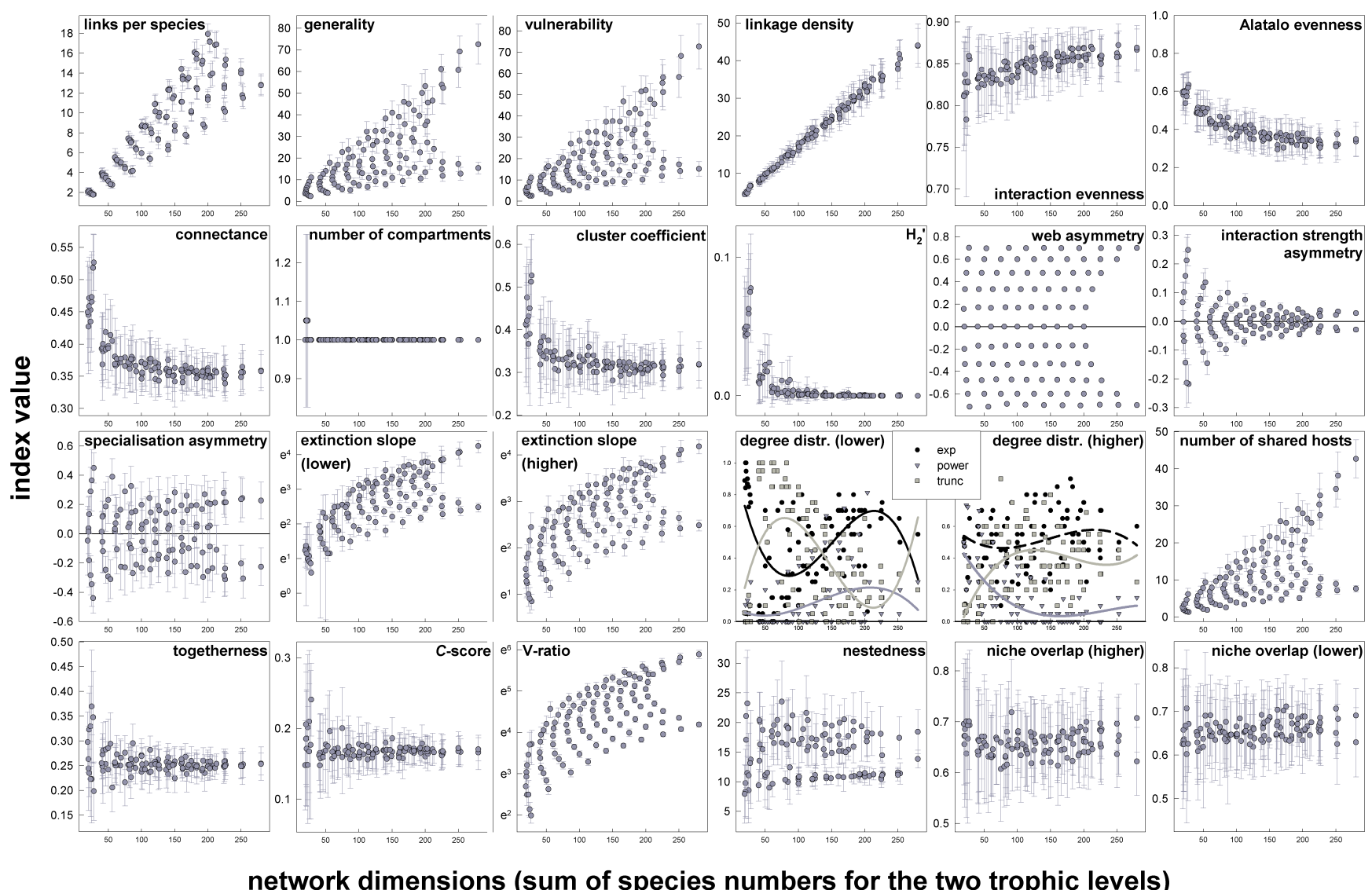

Fig. (2). Network dimension effects on index values. Indices insensitive to network dimensions can be interpreted at face value, while those sensitive require comparison with a null model. Lines in degree distribution plots are cubic regressions; dashed lines are not significant (i.e. $P>0.05$ ). Error bars are \pm 1 standard deviation of the mean (based on 20 replicates per datum). Note that $y$-axis is log-transformed for extinction slopes and V-ratio. Our setup limits the total number of species in both trophic levels to 280. Networks with many (>200) species in one trophic level will necessarily contain fewer in the other (visible as a "dent" at the right hand side in the web asymmetry scatter plot). This dent is also visible in several other network indices (e.g. generality, V-ratio), indicating that upper and lower limits of these indices are determined by the number of species in the higher and lower trophic level.

\section{Analyzing Networks}

The $(110+9) * 20 * 2=4760$ artificial networks, the 19 observed networks, the $1000 *(3+3) * 19=114000$ null and the perturbed networks were analyzed for the indices listed above. Means and standard deviations across replicates were calculated. For the fits of the degree distributions, we counted the proportion of replicates which were best fitted by the different fitting models. No formal statistical tests of simulated data were carried out, since given large enough sample sizes any effect will be significant. Instead, we interpret depicted trends conservatively.

\section{RESULTS}

\section{Changes in Network Indices: Simulated Networks}

Both network dimensions and sampling intensity affect many of the indices introduced above (Fig. 2 and 3). Most indices were systematically influenced by network dimensions, with the exceptions of number of compartments, $C$-score and niche overlaps. For interaction strength asymmetry, degree distribution fits and nestedness it was important which trophic level contained more species and network dimensions in general were not very conclusive.
Some indices were noticeably more sensitive to asymmetry in network dimensions when networks were small (less than 50 species in both levels together): interaction evenness, connectance, cluster coefficients, $\mathrm{H}_{2}{ }^{\prime}$, interaction strength asymmetry, togetherness and $C$-score. This suggests a minimum network size before there indices can be used with confidence.

The patterns were even clearer for the effects of sampling intensity (Fig. 3; see also the similar simulations of BanasekRichter et al. 2004). Only interaction strength and specialization asymmetry showed no clear response to sampling intensity, all other indices were substantially affected.

The observed networks contain, on average, 39\% ( $\pm 19 \%$ 1 standard deviation) of singleton interactions, i.e. pollinator species that were only once observed on a specific plant species (although there may be further records on other plant species). Excluding singletons had only minor effect on index values (usually less than $10 \%$ change), both for artificial and real networks (see Fig. S1). There were some indices however, for which singleton observations seemed very influential, in particular extinction slopes, the co- 


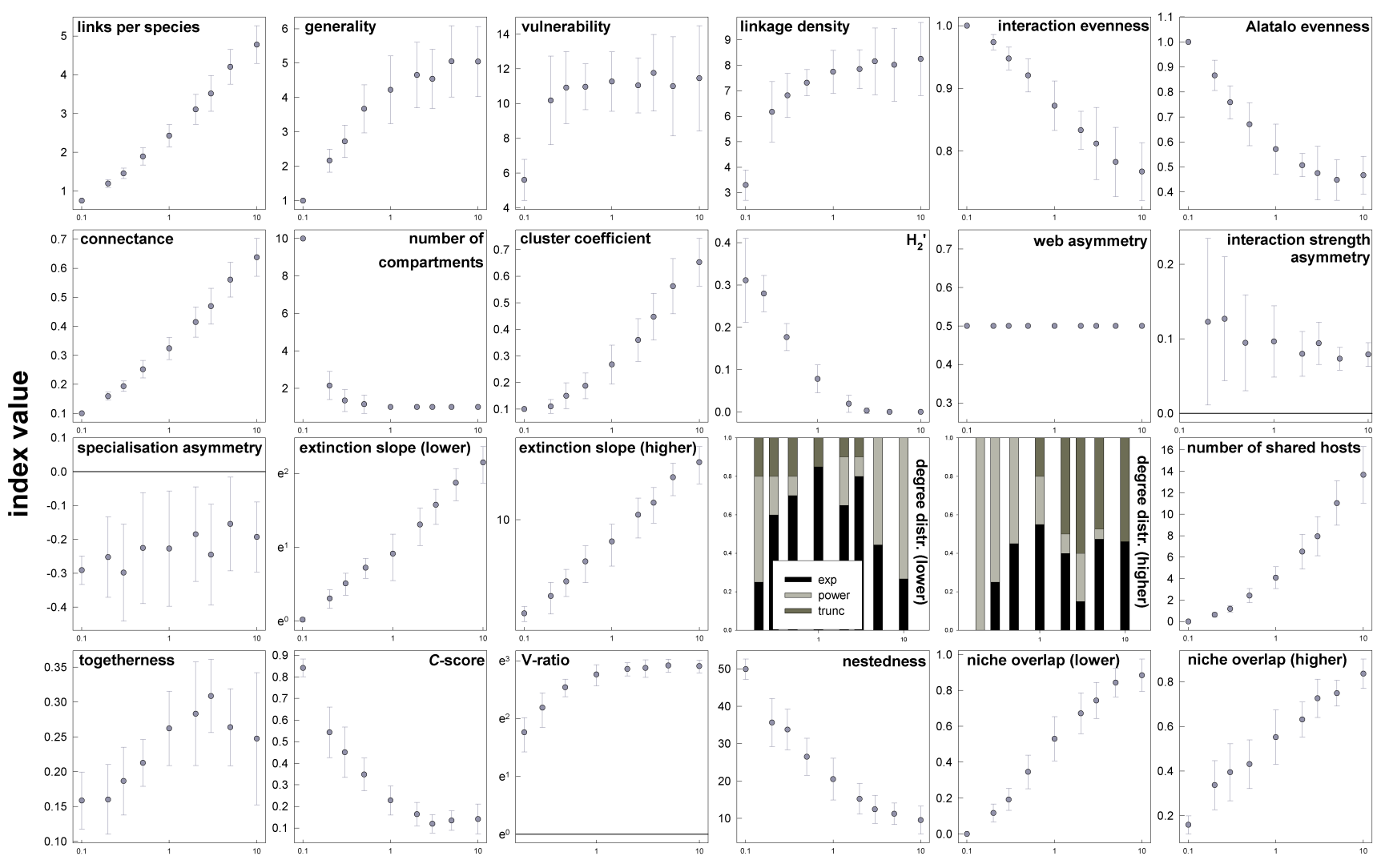

sampling intensity (mean number of observed interactions per species)

Fig. (3). Sampling intensity effects on index values. Error bars are \pm 1 SD based on 20 replicates. Reference lines indicate critical values (see index description). Degree distributions depict proportion of best fitting regression models for the 20 replicates at each level of sampling intensity. Note that $\mathrm{x}$-axis and some $\mathrm{y}$-axis were log-transformed.

occurrence measures togetherness and $C$-score, and nestedness. Overall, artificial and real networks responded consistently to removal of singletons.

Another way to investigate the robustness of network indices was to perturb the original matrix by randomly allocating an observed interaction to another cell in the network matrix ("re-wiring"). Different levels of re-wiring $(5,10$ or $20 \%)$ showed that most indices were little affected (Fig. S2). Most noticeably, links per species, connectance, interaction strength asymmetry and extinction slopes were altered by matrix perturbation.

The comparison of networks of different dimensions, different sampling intensity and the observed networks also reveals another point (Fig. S1): For most indices, network dimensions are crucial for reproducing the pattern observed in the real pollination networks, but sampling intensity is not: When network dimensions were varied widely, then index values are usually very different from those observed (first group of bars). Networks with the median dimensions of the observed webs but varying sampling intensity are usually rather similar to the observed networks (middle group of bars in Fig. S1). This suggests that null models correcting for the network dimensions are crucial, correction for sampling intensity are optional. However, there are exceptions from this pattern. Connectance, compartment structure, $\mathrm{H}_{2}$, , niche overlap and togetherness show moderate to pronounced deviations between observed and artificial networks.

\section{Changes in Network Indices: Real Networks and Null Models}

From the previous analysis of artificial webs we hypothesized that some pattern in observed indices may be explicable by the networks dimensions, and to a lesser extend by sampling intensity. The values for all 19 pollination networks plotted against network dimensions (Fig. S3) and sampling intensity (Fig. S4) are depicted in the appendix.

Fig. (4) shows a comparison of indices for the observed networks together with the three null models controlling for (1) network dimensions and marginal distributions (Patefield), (2) network dimensions and connectance (shuffle), and (3) network dimensions, interaction distribution and connectance (swap). Correlations between observed and null model means increased from Patefield $(|\mathrm{r}|=0.62 \pm 0.29$; Pearson correlation coefficient of null model vs. observed, averaged across all indices \pm 1 SD) over shuffle $(|\mathrm{r}|=0.74 \pm 0.27)$ to swap $(|\mathrm{r}|=0.80 \pm 0.25)$, indicating that constraints on connectance are more important than those on marginal totals. 


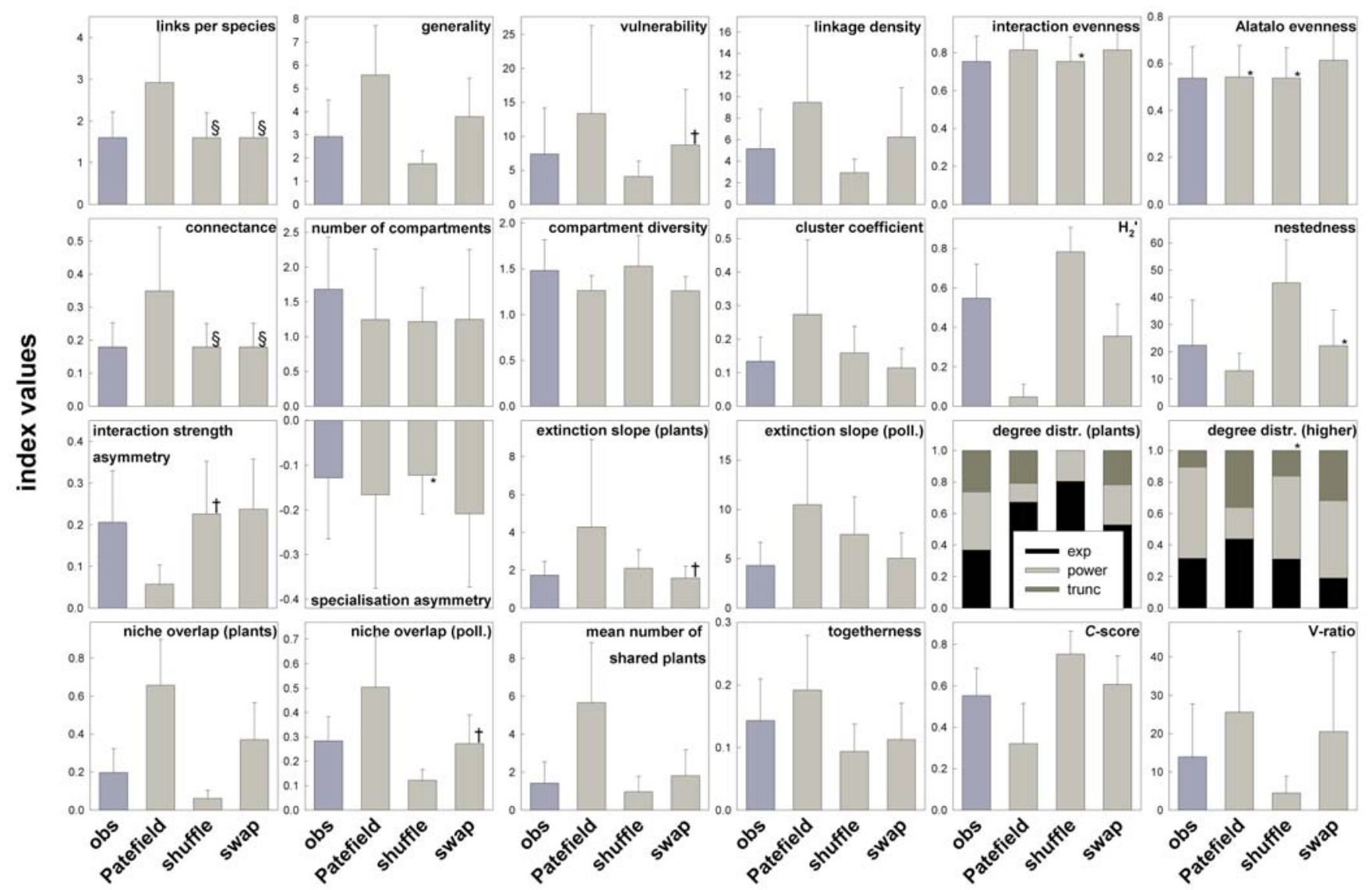

Fig. (4). Comparison of observed network (dark gray) properties and null model values (light gray). Based on 19 pollination networks and means of 1000 replicate null models for each observed network. Error bars are 1 standard deviation across the 19 networks; $\uparrow$ and $*$ indicate $P$-values of the power of a t-test of $<0.1$ and $<0.05$, respectively. In contrast to a t-test itself, its power indicates the probability that observed and null model are from different distributions. § indicates identity of values, due to constraints on null models.

\section{DISCUSSION}

\section{Sensitivity of Bipartite Network Indices to Network Dimensions, Dimensional Asymmetry, Sampling Intensity, Singletons and Errors}

Our simulations show that most indices are strongly affected by network dimensions and sampling intensity. The importance of network dimensions was previously documented for dependence asymmetry and connectance by Olesen and co-workers $(2002 ; 2006)$ and Blüthgen et al. (2007), who also showed that the $\mathrm{H}_{2}$ '-index was robust. For sampling intensity, and thus realized number of links, our results are concordant with the findings of Banasek-Richter et al. (2004) and Nielsen and Bascompte (2007), who report on moderate to strong effects. Blüthgen et al. (2006) report on strong effects of the number of interactions on connectance, while their proposed $\mathrm{H}_{2}$ ' was unaffected. For the biogeographic indices $C$-score and nestedness, Ulrich and Gotelli (2007a) showed an effect of matrix filling, but also of the distribution shape of marginal abundances. Simulation studies suggest that a high proportion of truly occurring interactions need to be recorded to accurately describe the network (Novak and Wootton 2008).

The exclusion of singleton observations has not been analyzed previously for networks, while it has received considerable attention in multivariate analysis of community data (Jongman et al. 1995; Legendre and Legendre 1998; McGarigal et al. 2000; McCune et al. 2002). Our simulations indicate that the removal of singleton observations had generally little effect, neither for artificially generated networks nor for observed networks. This is somewhat surprising, given the high proportion of singleton observations (around 40\%) and the fact that they may heavily dominate some indices (see, e.g., the discussion of singleton species (not singleton observations) and their effect on dependence asymmetry in Blüthgen et al. 2007). For qualitative indices this insensitivity to singleton observations gives evidence of a random distribution of rare interactions. For qualitative indices however, it suggests domination by cells with high interactions values. If true, the flip side of this interpretation is a low sensitivity to changes in any cell apart from those with high values. An alternative explanation is that rare species do not contribute substantially to secondorder patterns, even questioning their ecological importance in pollination networks. Further research is needed to investigate the sensitivity of different indices to changes in species specialization and their interactions.

Similarly, error in the form of noise (caused for example by typos and misidentification) led to very little bias in network indices (Fig. S2). Only interaction strength 
asymmetry and extinction slopes were noticeably affected. In a multi-year study of pollination webs, Petanidou et al. (2008) reports on fluctuations in network indices larger than those introduced in our perturbation simulations. They attribute this variance to a high level of species turnover and less to specialization flexibility, which is the component our perturbation approach tests.
Overall, our simulations indicate a definite need for a careful interpretation of absolute index values. Following the pledges by Gotelli and Graves (1996), we strongly encourage the use of null models to provide a baseline for expectations based on non-informative network properties alone (see also Vázquez and Aizen 2003, 2006). Before addressing some current hypotheses on network structure by

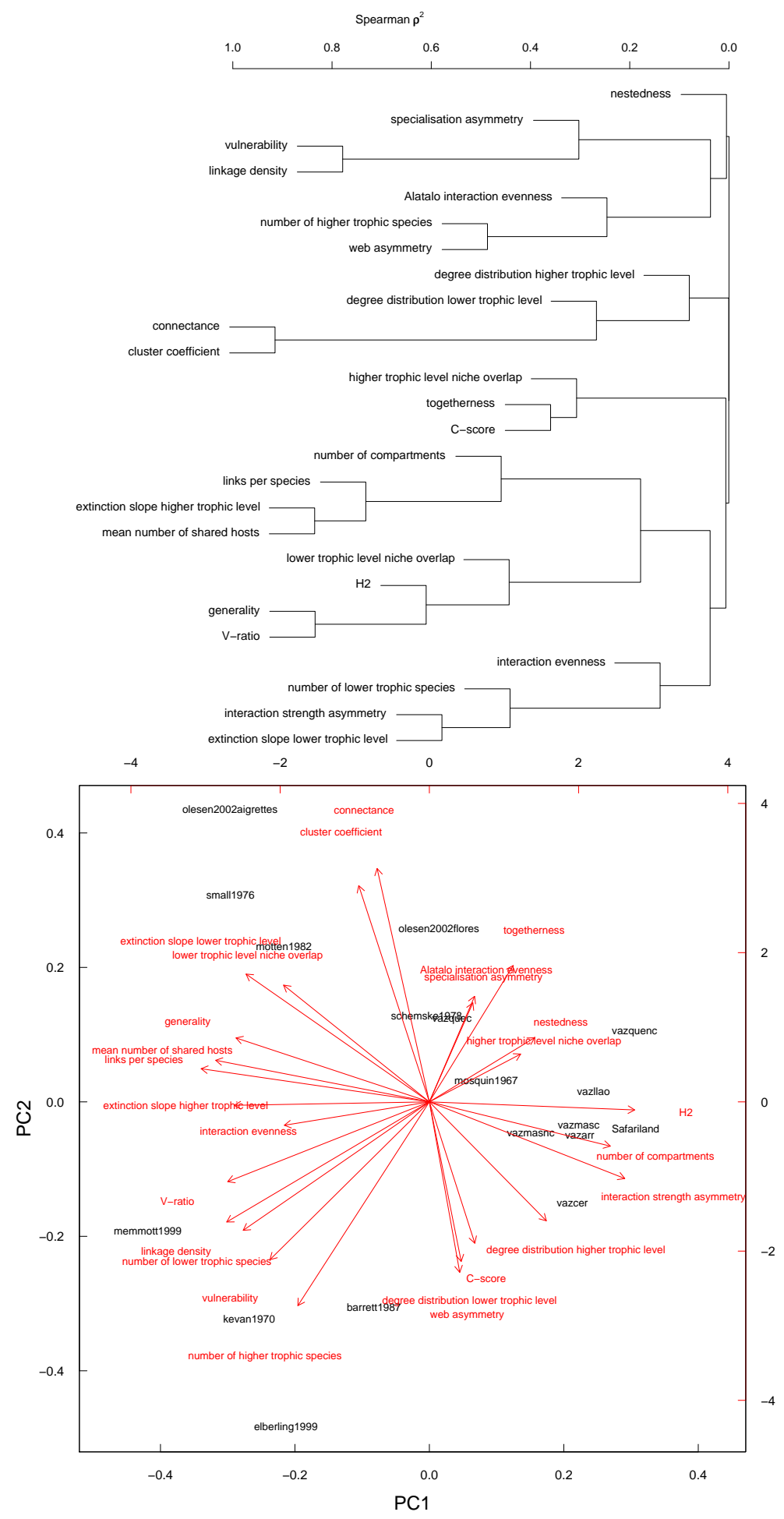

Fig. (5). Relationship between indices of the observed pollination networks as depicted in a cluster analysis (top: distance based on squared Spearman's rank correlation coefficient) and a PCA (bottom). These two-dimensional representations hide the fact that variables plotted here as distant may well be correlated in other dimensions. In both cases the large network of Kato et al. (1990) was omitted, because it distorts the presentation (although not the outcome: see Fig. (S5)). 
means of null models, we investigate patterns among indices in the following section.

\section{Interrelationship between Indices}

The high correlation between connectance and several other indices (see Fig. (3) for simulated and Table S1 for observed networks) may have two causes: either connectance is a critical feature of networks, or the network indices depicted mostly measure the same property as connectance. The latter option is a re-wording of the claim that the various different indices really all measure one of two network features: dimensionality (i.e. number of species in various forms) or specialization of species (which is also related to connectance). By performing a cluster and a principle component analysis (PCA), we grouped network indices based on their (standardized) values for the observed pollination networks (Fig. 5). In the PCA, 78\% of the total variance is captured by the first axis, which is dominated by the effect of number of higher level species. The second, much less important axis (18\%) is driven by the number of lower trophic level species, V-ratio and generality. This result alone indicates that network dimensions are of fundamental influence on virtually all network indices. The cluster analysis additionally depicts significant correlations between several indices (i.e. high correlation between two or more indices). Fig. (5) shows that vulnerability and linkage density essentially measure the same property; so do connectance and cluster coefficient; and higher trophic level niche overlap and $C$-score (see also table $\mathrm{S} 1$ for all correlations). Only very few indices provide independent network descriptions, among them specialization asymmetry, nestedness and (Alatalo's) interaction evenness.

Finally it is noteworthy that the response of pollination networks to random extinctions is highly correlated with the mean number of shared hosts (for the higher trophic level) and the number of lower trophic level species (for the lower trophic level). That simple network indices are highly correlated with extinction slopes has probably more to do with the simulation of extinction events than with the networks true ability to buffer pollinator loss: since we have no information on likely extinction sequences we simulated a random extinction sequence. Also, our correlative analysis does not support the view that "patterning of IS [interaction strength] is essential to maintaining community persistence" (Garcia-Domingo and Saldaña 2008, p. 342). Assuming that community persistence can be measured by extinction slopes and that interaction strengths find their representation in generality, interaction evenness or interaction strength asymmetry, no close relationship between these latter variables and extinction slopes was evident from the cluster analysis (Fig. 5).

\section{Testing Specific Patterns with Pollination Webs and Null Models}

Our analysis above shows that some network indices are strongly affected by first-order properties of the network, such as number of species and their respective abundance. Second-order properties, such as connectance or nestedness, are hence to some extent results of first-order characteristics. Following the logic of Occam's razor, interaction patterns that do not exceed the descriptive power of simpler, firstorder properties, may not represent a meaningful alternative explanation. In this section we explore to which extent firstand second-order properties are required to reproduce observed values of several established paradigms about properties of ecological networks by comparing observed quantitative pollination networks with three null models (Patefield $=$ constant marginal sums; shuffle $=$ constant connectance; and swap = constant marginal sums and constant connectance). The comparison of observed values and null model predictions across all networks is depicted in Fig. (4) (as mean values $\pm \mathrm{sd}$ ). However, averages across all 19 pollination networks may give an incomplete and even misleading picture, since underestimation of high and overestimation of low values may well yield similar averages for observed and null model. Therefore, we here use calibration plots of null model versus observed index values for each network as our key statistic.

\section{Pattern 1: In Real Pollination Webs, Interactions have Degree Distributions following a (Truncated) Power Law, Reflecting Ecological Adaptation}

Pattern in degree distributions have been proposed as presenting information on the structure of ecological networks (Dunne et al. 2002; Jordano et al. 2003; Montoya et al. 2006; Sun et al. 2006). Whatever the possible ecological interpretation of this phenomenon, the 19 pollination networks support previous studies claiming (truncated) power law distribution of species degrees to be prevalent among ecological networks (but see Burns 2007).

Fig. (6) (bottom panels) shows that exponential and power fits were observed in seven networks each, but only exponential fits were predicted well by null models I and III. All null models failed to accurately predict the power and truncated power function fits. Overall, null model III had the highest success in reproducing the observed patterns in degree distributions: Fisher's sign test found no significant difference between null model III and observed fits (74\% correct: $P=0.168)$. Null models I and II were significantly different from observed (49 and 33\% correct fits: $P<0.01$ and $<0.001$, respectively). This means that first-order effects alone (i.e. species abundances) are insufficient to reproduce the observed degree distribution pattern.

For pollinators a similar pattern emerged (Fig. 6, top panels), although here null model II was more successful than for plants. 41, 64 and $70 \%$ of null models were correct (Fisher's test for significance of difference between null and observed: $P<0.001, P=0.9035$ and $P=0.3515$, for null model I, II and III, respectively).

\section{Conclusion}

Degree distributions cannot be reproduced without taking second-order properties (in this case connectance) into account. Since connectance has been argued to be in itself the result of co-evolutionary processes (Petanidou and Potts 2006), we tentatively conclude that also power law-type degree distributions may have evolutionary origin.

\section{Pattern 2: Pollination Network Display Asymmetry in their Dependencies: Specialists Interact more with Generalists and vice versa}

In the evolution of specialization, pollinators that became specialised on a rare plant species will have been selected 


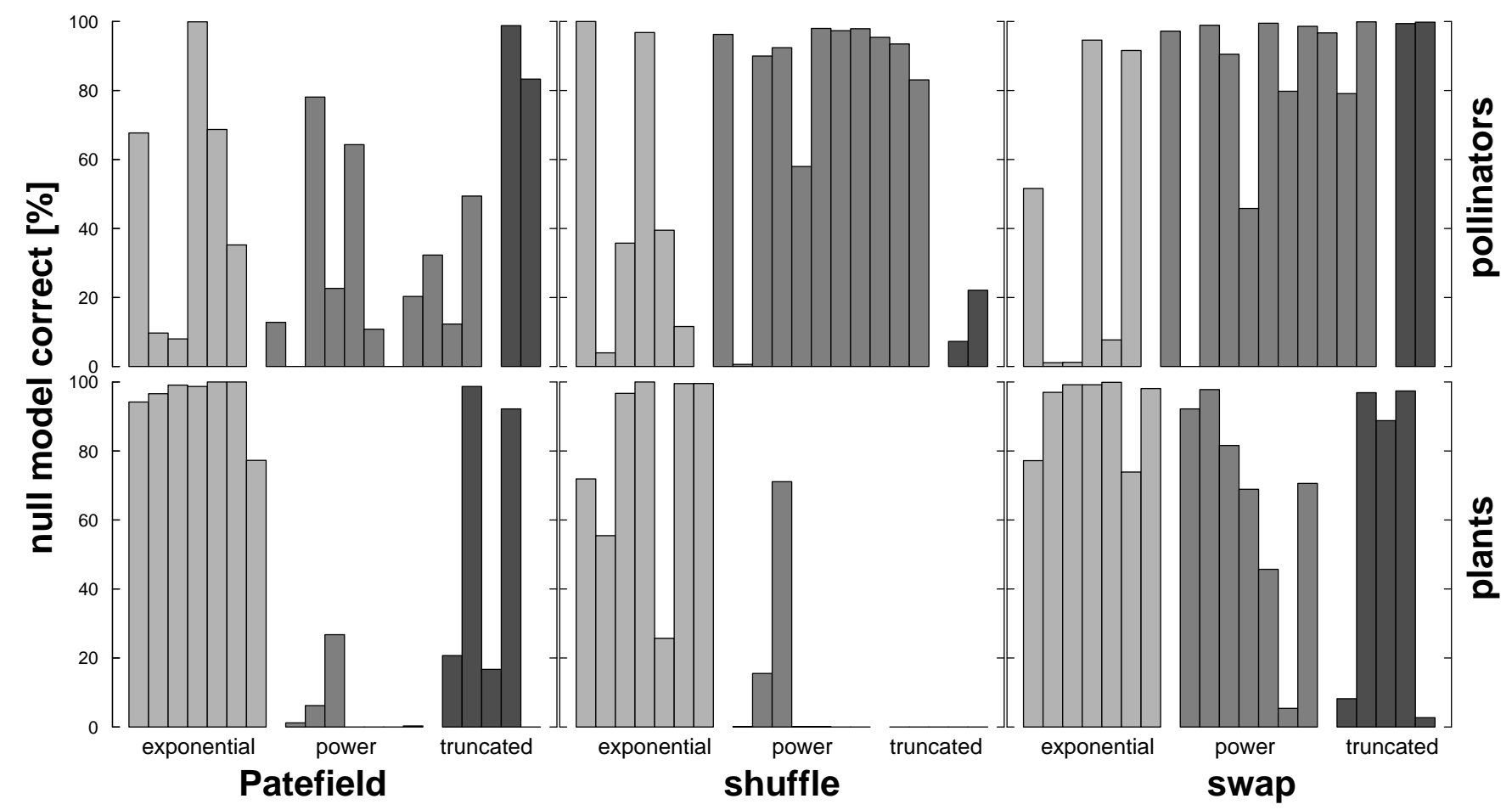

Fig. (6). Results of the three null models for degree distributions in pollination networks. Each panel depicts the percentage (of 1000 replicate runs) of null models that yielded the same degree distribution as the observed. Observed networks were best fitted by either exponential (light gray), power function (middle gray) and truncated power function (dark gray). Each network is represented by one bar, top row are pollinators, bottom row plants. See text for statistics and Fig. (4) for a summary across all 19 networks.

against by population fluctuations of their hosts. Hence, we would expect extant pollinators to be specialised on very common species (Vázquez and Aizen 2006). On the other hand, rare plant species benefit most from attracting many different pollinators. As a consequence, Jordano (1987), Vázquez and Simberloff (2002) and Bascompte et al. (2006) proposed that plants and pollinators show asymmetry in their dependence on the mutualist, with generalist pollinators having specialist host plants, and vice versa. From an evolutionary perspective, dependence asymmetry is very interesting (Tylianakis 2008), because it depicts the net effect of a long-term evolutionary adaptation process. Their findings have received criticism, both for the statistic employed (Blüthgen et al. 2007; Vázquez et al. 2007) and the population consequences claimed (Holland et al. 2006).

Our null models explain large proportions of the variance in observed dependence asymmetry (Pearson's correlation coefficient of $r=0.80$ for all three null models). Calibration plots indicate null model II underestimates the observed pattern, while null model I and III replicate the observed pattern well (Fig. (7), see also Fig. (4) "Interaction strength asymmetry", for a different result, where null models II and
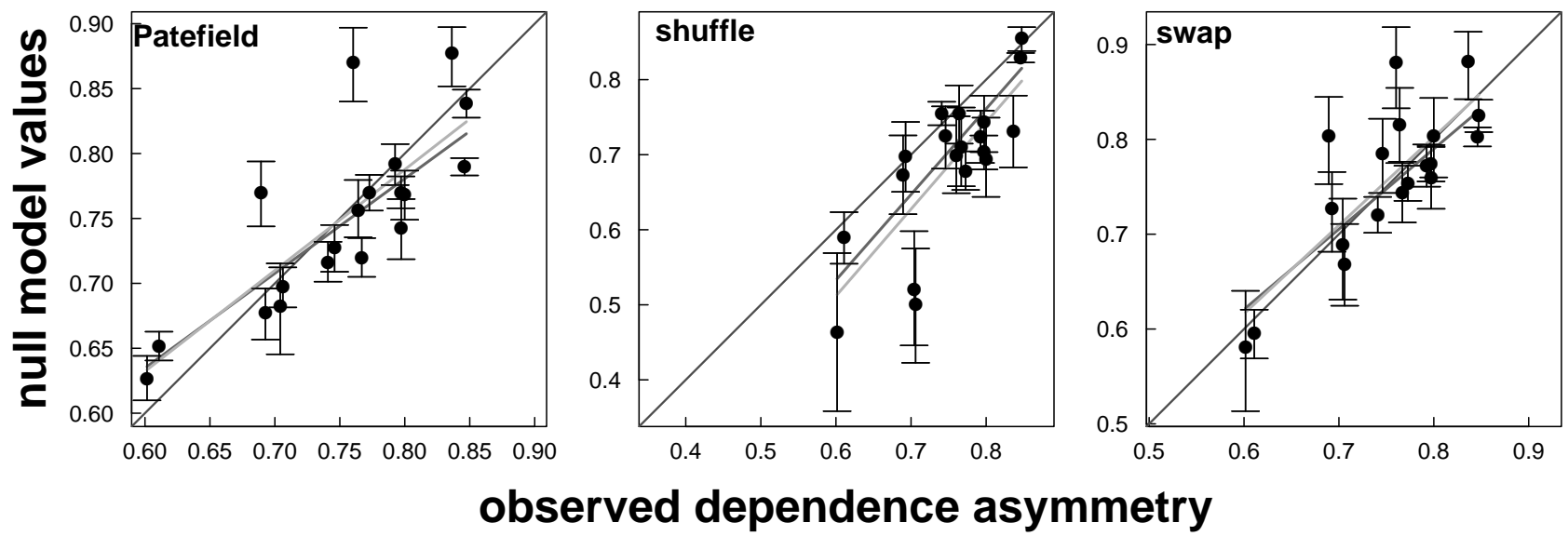

Fig. (7). Calibration plot for the three null models against observed dependence asymmetry. Diagonal represents a perfect reproduction of the observed values by the null model. Error bars are $95 \%$ confidence intervals of the 1000 replicates. Regressions are weighted by the reciprocal of the standard deviation of the null models (dark gray) or unweighted (light gray), and are hatched when not significant. Average distance between unweighted regression and diagonal is equivalent to differences between bars in Fig. (4). $R^{2}$-values for weighted regressions are $0.71,0.67$ and 0.64 , respectively. 
III are reproducing the pattern in the improved measure "interaction strength asymmetry"). The lack of difference in fit between null models I and III indicates that second-order properties are of minor importance for reproducing dependence asymmetry.

\section{Conclusion}

Observed patterns of dependence asymmetry could be reproduced based on species abundances alone, with a slight improvement after constraining for connectance. Hence, we conclude that first-order effects explain most of the dependence asymmetry observed in pollination networks, but that connectance adds additional fine tuning.

\section{Pattern 3: Ecological Communities are Nested}

Pollination networks have been described as being nested (Bascompte et al. 2003; Santamaría and Rodríguez-Gironés 2007). In nested networks, pollinator communities on plants with few pollinators are subsets of plants with more pollinators. This measure was transferred from biogeography, where it is used to describe e.g. species assemblages on different-sized islands. It has also been employed in other ecological communities (Gotelli and Graves 1996; Brualdi and Sanderson 1999; Rohde 2001; Gotelli and Rohde 2002; Almeida-Neto et al. 2007). Recently, nestedness has been found within network compartments and modules, rather than across the entire network (Lewinsohn et al. 2006; Olesen et al. 2007). Previous null model analyses indicated that nestedness is a consequence of marginal distribution (Burns 2007; Blüthgen et al. 2008), which are often lognormal (Blüthgen et al. 2008).

Null model I (Patefield) constructs networks with higher nesting (and hence lower temperature) than observed (Fig. (8); Pearson's $r=0.27)$. The contrary is true for null model II (shuffle), which is consistently less nested (= higher nestedness temperature) than the observed networks, but in a similar sequence $(r=0.85)$. Null model III (swap) generated networks with nestedness temperature very similar to those observed $(\mathrm{r}=0.87)$.

\section{Conclusion}

Nestedness as such can easily be produced by null model I. However, connectance needs to be taken into account to yield networks similar to those observed. We thus confirm the specific nestedness temperature as a property of the network rather than of first-order properties alone.

\section{Pattern 4: Specialization-Induced Separation of Species is Rare Compared to Positive Associations among Species}

Pollinator specialization has seen a paradigm shift over the last several decades. Previously heralded as a showcase for co-evolution by ecologists (Darwin 1862), evidence has now accumulated that shows strict specialization to be the exception rather than the rule (Waser et al. 1996; Waser 2006). Also other communities display more often positive associations than specialization-induced complementarity (Rohde 2001; Gotelli and Rohde 2002). In the context of pollination networks this could mean that plants are similarly attractive to pollinators, even though pollinators may slightly differ in their preferences. Still, specialization may be higher than in null models (Vázquez and Aizen 2003).

Using the $C$-score as a measure of non co-occurrence, complementarity or "dis-occurrence", we find observed levels of dis-occurrence to be significantly higher than predicted by marginal totals (points are below the calibration line in Fig. (9), upper left panel), but lower than predicted by connectance (points are above the line; upper middle panel). Again, constraining the null model both by marginal sums and connectance leads to the closest reproduction of observed values (null model III: swap). The correlation between observed and null model values is moderate to high for Patefield, shuffle and swap (Pearson's $r=0.75,0.68$ and 0.81 , respectively), indicating that these algorithms only capture parts of the structuring mechanisms.

A similar picture emerged for the network-wide specialization index $\mathrm{H}_{2}$ ' (Fig. 9, lower panels). Again, none

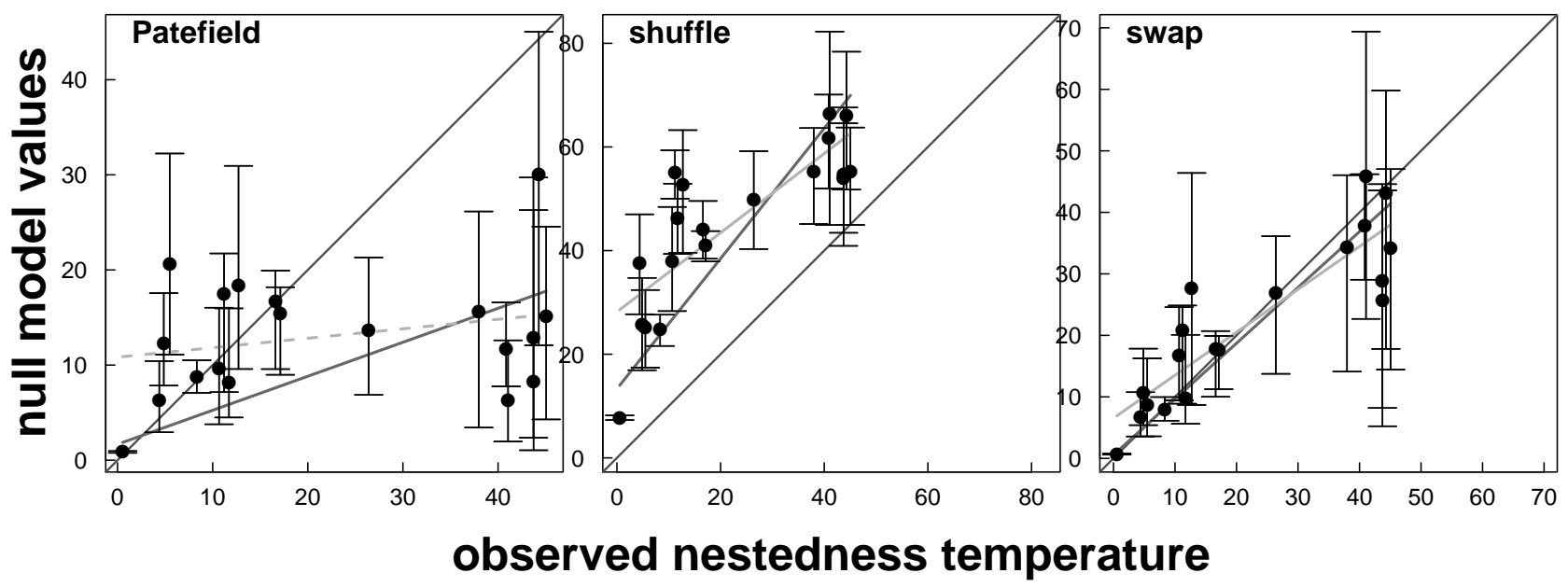

Fig. (8). Calibration plot for the three null models against observed network nestedness temperature (the lower the temperature, the more nested). Diagonal represents a perfect reproduction of the observed values by the null model. Error bars are 95\% confidence intervals of the 1000 replicates. Regressions are weighted by the reciprocal of the standard deviation of the null models (dark gray) or unweighted (light gray), and are hatched when not significant. Average distance between unweighted regression and diagonal is equivalent to differences between bars in Fig. (4). $R^{2}$-values for weighted regressions are $0.49,0.73$ and 0.92 , respectively. 

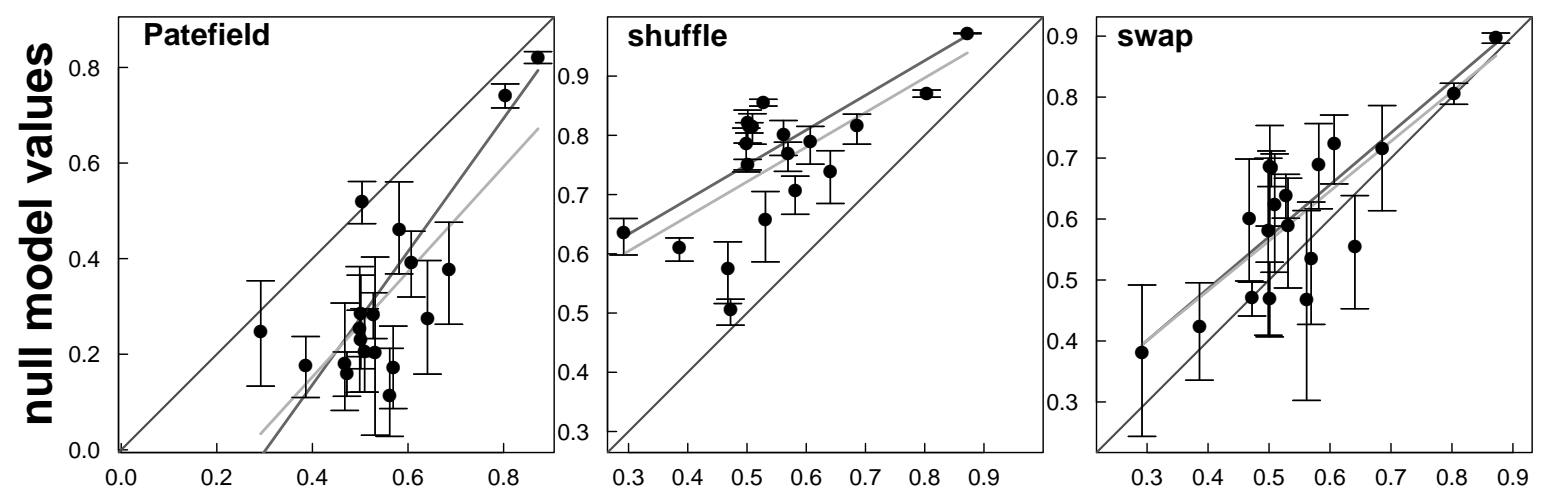

observed C-score

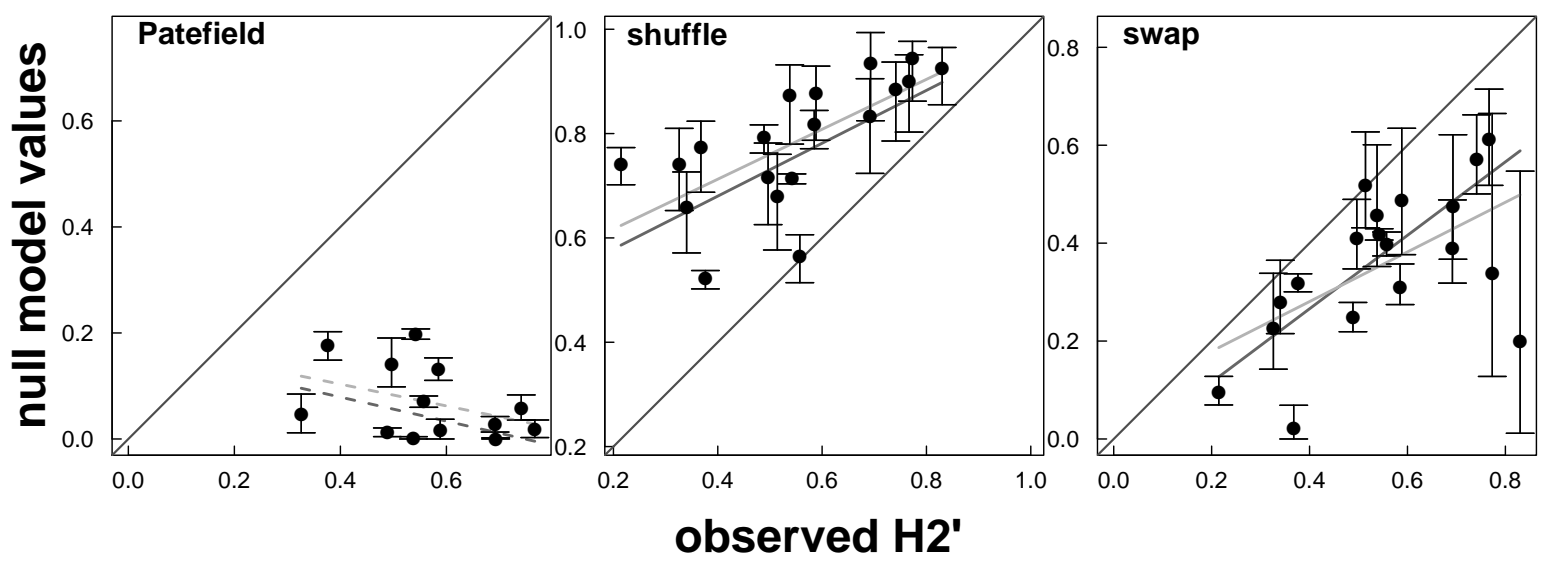

Fig. (9). Calibration plot for the three null models against observed co-occurrence ( $C$-score, upper row) and network-wide specialization $\left(\mathrm{H}_{2}\right.$, lower row). Diagonal represents a perfect reproduction of the observed values by the null model. Error bars are $95 \%$ confidence intervals of the 1000 replicates. Regressions are weighted by the reciprocal of the standard deviation of the null models (dark gray) or unweighted (light gray), and are hatched when not significant. Average distance between unweighted regression and diagonal is equivalent to differences between bars in Fig. (4). $R^{2}$-values for weighted regressions are for $0.81,0.79$ and 0.84 for $C$-scores, and $0.07,0.34$ and 0.54 for $\mathrm{H}_{2}$ '.

of the null models fully captured the pattern observed, and correlations were absent to high. The poor correlation between null model I and observed must be attributed to the fact that $\mathrm{H}_{2}$ ' was designed to correct for species abundance, and hence its values should be unaffected by null model I.

Specialization and separation are also the basis of dependence asymmetry discussed under pattern 2 (Fig. 7). Clearly, these indices are conceptually related, but differ in their focus on single trophic levels $\left(\mathrm{H}_{2}\right.$, and C-score) or the relationship between both trophic levels (DA). As a consequence, their values are not correlated (DA vs. $\mathrm{H}_{2}$ ': r=0.04; DA vs. $C$-score: $0.05 ; H_{2}$ vs. $C$-score: 0.24 , all $P>$ $0.3)$.

\section{Conclusion}

Specialization, and hence dis-occurrence, is more common than would be expected from random pairing of species, but less than the observed connectance would suggest. Moderate network-wide specialization seems to be pervasive in pollination networks.

\section{Pattern 5: Real Webs are more Robust to Extinction than Random Webs}

Members of ecological networks are under constant evolutionary pressure. Fluctuations in species abundance are likely to purge stiff connections from the network, in favor of weaker, more diffuse interactions (Berlow 1999; Berlow et al. 2004). Hence, we may expect real networks to be more stable to extinction of species than random webs (Memmott et al. 2004; Srinivasan et al. 2007). On the other hand, any step towards specialization makes a pollinator more vulnerable to the extinction of its forage plant. Hence, pollination networks that show specialization may also show reduced stability to extinctions.

Our null models I and II overestimate extinction slopes for secondary plant extinctions (Fig. 10 lower panels), i.e. plants in observed networks go extinct sooner than would be expected on the basis of marginal distributions or connectance alone. Only null model III, with its constraints on marginal sums and connectance, has extinction slopes slightly lower than those of the observed network. Similarly, secondary extinction slopes for pollinators are overestimated, meaning that observed networks are less robust to random extinctions than would be expected under null model assumptions. This pattern seems to be a logical consequence of specialization in networks: any increase in specialization will necessarily lead to a higher vulnerability to extinction of the mutualist (Pearson's correlation between slopes and specialization index $\mathrm{H}_{2}{ }^{\prime}: r=-0.41, P=0.077$ for plants and $r=-0.48, P=0.036$ for pollinators: higher specialization 


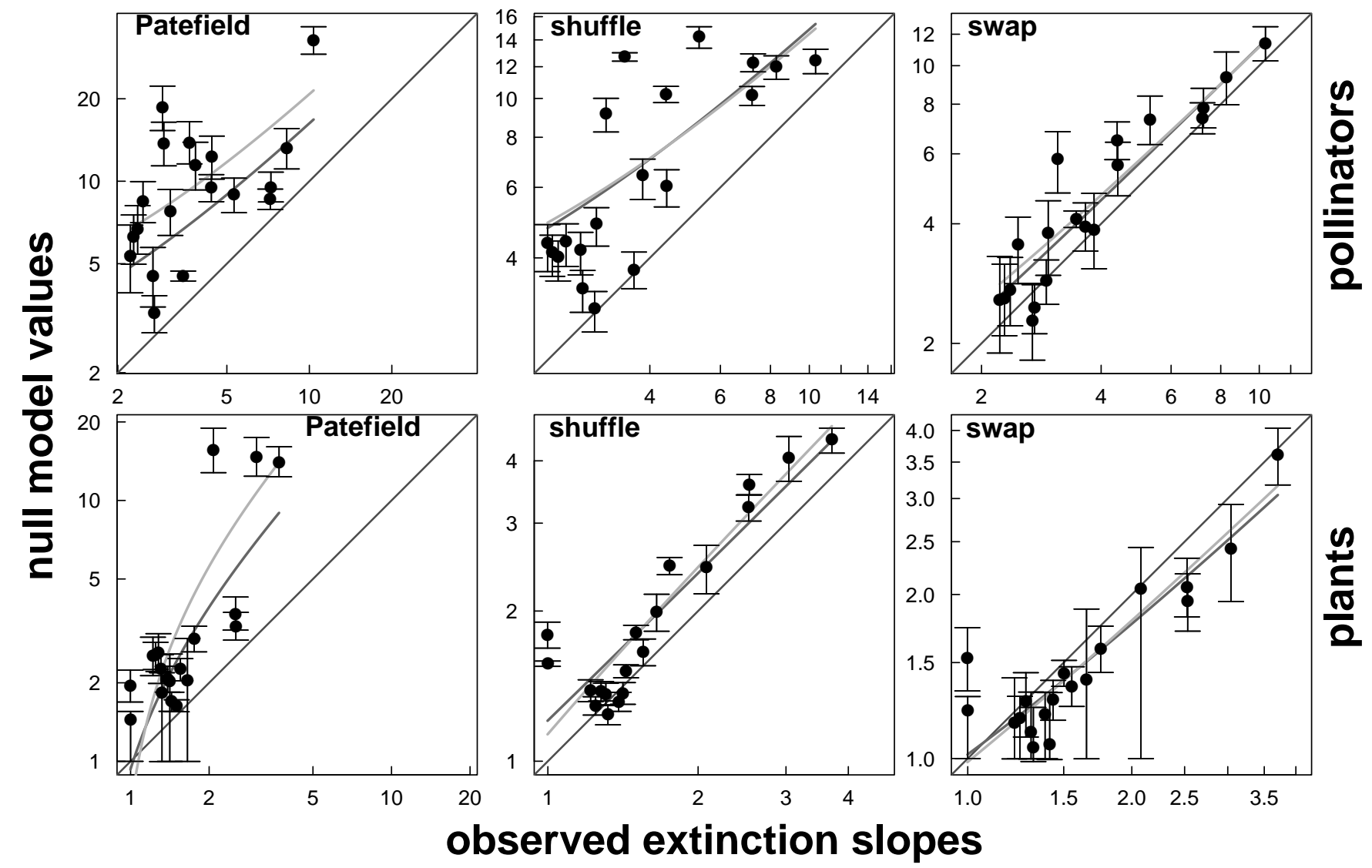

Fig. (10). Calibration plot for the three null models against extinction slopes for the observed networks (random loss of plants and pollinators, upper and lower panels, respectively). Diagonal represents a perfect reproduction of the observed values by the null model, points above the line indicate higher robustness of null model networks. Error bars are $95 \%$ confidence intervals of the 1000 replicates. Regressions are weighted by the reciprocal of the standard deviation of the null models (dark gray) or unweighted (light gray), and are hatched when not significant. Average distance between unweighted regression and diagonal is equivalent to differences between bars in Fig. (4). $R^{2}$-values for weighted regressions are for $0.30,0.44$ and 0.89 for pollinators, and $0.41,0.75$ and 0.81 for plants. Note that both axes are logarithmically scaled, leading to curvi-linear regression lines.

leads to shallower extinction slopes, i.e. earlier secondary extinctions).

\section{Conclusion}

In contrast to the paradigm of more-stable-than-randomnetworks, plants and pollinators in real pollination networks are less robust to extinction than those in our null models.

Overall, our test of current network pattern paradigms yields two general results. Firstly, although most of the tested patterns (degree distributions for plants (but not for pollinators); dependence asymmetry; specialization and cooccurrence) are not reproducible fully by our null models, a substantial part of their pattern can be, at least across many networks (Fig. 4). Null models usually are moderately to highly correlated with observations, indicating that network dimensions and marginal distributions (i.e. first-order descriptions of the network) contribute substantially to network patterns (second-order descriptions). In practice this means that we should attempt to subtract these first-order influences from network description before we start interpreting processes at the network level. In addition, some network pattern are at best uninformative (dependence asymmetry can be predicted largely from the marginal distributions of the network: Fig. 8) or even opposing current ecological view (null model networks are more stable under random extinction than observed networks: Fig. 10).

Secondly, our null model III ("swap"), which is constrained by first-order properties (abundance distribution of network participants) and second-order properties (network connectance), overall yields very high agreement between observation and null model. This indicates that the role of connectance, one of the very first and most simple network descriptors (Pimm 1982; Jordano 1987), is possibly still crucial to furthering our understanding of the ecology and evolution of bipartite network. In how far other secondorder indices are required for further fine-tuning of null model III remains to be seen.

\section{Future Directions in Bipartite Network Analysis}

In this study we have highlighted both potentials and problems of bipartite network analysis. The plethora of largely correlated network indices makes communication between analysts more difficult, since each of them may have his or her favorite index. Our analysis software at least allows the calculation of all these indices and visualizes the bipartite networks, even if there will rarely be the journal space to depict them. While over time certainly several more 
indices will be developed and incorporated, these are likely to be tailored to specific questions. To date, a seemingly simple question such as "How specialised are pollinators?" (Petanidou and Potts 2006) can be addressed by several different network indices, both at the individual level (using a species' degree or $d$-value: see function specieslevel in bipartite) and at the network level (e.g. using the indices $\mathrm{H}_{2}$, $C$-score, niche overlap). Our analysis of their interrelationship indicates that they are likely to deliver qualitatively similar results.

The litmus test for our understanding of the mechanisms structuring networks is their reproduction by simple algorithmic rules (null models, pattern-oriented modeling, and inverse modeling). The three null models presented here are only first steps on the way towards more complex rules that may eventually reproduce patterns observed in real networks. By comparing nested null models (such as the Patefield and the swap algorithm) we shall be able to elucidate the role the new feature plays.

In our real data analysis we focused on pollination networks, but host-parasitoid networks are almost as intensively studied (see, e.g., Müller et al. 1999a; Morris et al. 2004; Tylianakis et al. 2007). We expect these networks to differ fundamentally (Vázquez et al. 2007), since antagonistic interactions should exert stronger evolutionary pressures and depict a higher level of specialization (Abrams 2000; Berlow et al. 2004). Hence, potential pattern need to be interpreted considering the purpose of each index, since indices derived with antagonistic interactions in mind (such as generality, vulnerability and niche overlap) may be less suitable for mutualistic networks.

Finally, Bascompte et al. (2006), building on earlier concepts of May (1973) and Pimm (1982), propose to investigate the consequences of observed network pattern for the population dynamic of its participants. Predictions thus generated and validated against field data would provide a strong test of our ability to infer processes from patterns (Gaston and Blackburn 2000; Ings et al. 2008).

\section{ACKNOWLEDGEMENTS}

We are grateful to Miguel Rodríguez-Gironés for sharing his nestedness code with us and to various pre-release testers for reporting errors in bipartite. Two anonymous reviewers improved clarity in form and content of a previous version. CFD and BG acknowledge funding by the Helmholtz Association (VH-NG-247) and JF by the German Federal Environmental Foundation (AZ 20007/915).

\section{SUPPLEMENTARY MATERIAL}

Supplementary material can be viewed at www.bentham. org/open/toecolj.

\section{REFERENCES}

Abrams, PA (2000) The evolution of predator-prey interactions: theory \& evidence. Annual Review of Ecology \& Systematics, 31, 79-105.

Alatalo, RV (1981) Problems in the measurement of evenness in ecology Oikos, 37, 199-204.

Allesina, S, Alonso, D \& Pascual, M (2008) A general model for food web structure. Science, 320, 658-60.
Almeida-Neto, M, Guimarães, P, Guimarães-Jr, PR, Loyola, RD \& Ulrich, W (2008) A consistent metric for nestedness analysis in ecological systems: reconciling concept \& measurement. Oikos, 117, 1227-39.

Almeida-Neto, M, Guimarães, PR \& Lewinsohn, TM (2007) On nestedness analyses: rethinking matrix temperature \& anti-nestedness. Oikos, $116,716-22$

Atmar, W \& Patterson, BD (1993) The measure of order \& disorder in the distribution of species in fragmented habitat. Oecologia, 96, 37382.

Banasek-Richter, C, Cattin M-F \& Bersier, LF (2004) Sampling effects \& the robustness of quantitative $\&$ qualitative food-web descriptors. Journal of Theoretical Biology, 226, 23-32.

Bascompte, J, Jordano, P, Melian, CJ \& Olesen, JM (2003) The nested assembly of plant-animal mutualistic networks. Proceedings of the National Academy of Sciences of the United States of America, 100, 9383-7.

Bascompte, J, Jordano, P \& Olesen, JM (2006) Asymmetric coevolutionary networks facilitate biodiversity maintenance. Science, 312, 431-3.

Batagelj, V \& Mrvar, A (2003) In: Jünger, M \& Mutzel, P Eds. Pajek analysis \& visualization of large networks. Graph Drawing Software, Springer, Berlin, pp. 77-103.

Berlow, EL (1999) Strong effects of weak interactions in ecological communities. Nature, 398, 330-3.

Berlow, EL, Neutel, AM, Cohen, JE, de Ruiter, PC, Ebenman, B, Emmerson, M, Fox, JW, Jansen, VAA, Jones, JI, Kokkoris, GD, Logofet, DO, McKane, AJ, Montoya, JM \& Petchey, O (2004) Interaction strengths in food webs: issues \& opportunities. Journal of Animal Ecology, 73, 585-98.

Bersier, LF, Banasek-Richter, C \& Cattin, MF (2002) Quantitative descriptors of food-web matrices. Ecology, 83, 2394-407.

Blüthgen, N, Fründ, J, Vázquez, DP \& Menzel, F (2008) What do interaction network metrics tell us about specialization $\&$ biological traits? Ecology, 89, 3387-99.

Blüthgen, N, Menzel, F \& Blüthgen, N (2006) Measuring specialization in species interaction networks. BMC Ecology, 6, 12

Blüthgen, N, Menzel, F, Hovestadt, T, Fiala, B \& Blüthgen, N (2007) Specialization, constraints \& conflicting interests in mutualistic networks. Current Biology, 17, 1-6.

Brualdi, RA \& Sanderson, JG (1999) Nested species subsets, gaps, \& discrepancy. Oecologia, 119, 256-64.

Burns, KC (2007) Network properties of an epiphyte metacommunity. Journal of Ecology, 95, 1142-51.

Clauset, A, Moore, C \& Newman, MEJ (2008) Hierarchical structure \& the prediction of missing links in networks. Nature, 453, 98-101.

Darwin, CR (1862) On the Various Contrivances by which British \& Foreign Orchids are Fertilised by Insects, \& on the Good Effects of Intercrossing. John Murray, London.

Diamond, JM (1975) Assembly of species communities. In: Cody, M \& Diamond, JM Eds. Ecology \& Evolution of Communities, Belknap Press, Harvard, MA, 342-444.

Diamond, JM \& Case, TJ (1986) Community Ecology. Harper Row, New York.

Dicks, LV, Corbet, SA \& Pywell, RF (2002) Compartmentalization in plant-insect flower visitor webs. Journal of Animal Ecology, 71 32-43.

Dormann, CF, Gruber, B \& Fründ, J (2008) Introducing the bipartite package: analysing ecological networks. $R$ News, 8, 8-11.

Dunne, JA, Williams, RJ \& Martinez, ND (2002) Food-web structure \& network theory: the role of connectance \& size. Proceedings of the National Academy of Science USA, 99, 12917-22.

Fortuna, MA \& Bascompte, J (2006) Habitat loss \& the structure of plantanimal mutualistic networks. Ecology Letters, 9, 281-6.

Garcia-Domingo, JL \& Saldaña, J (2008) Effects of heterogeneous interaction strengths on food web complexity. Oikos, 117, 333-43.

Gaston, KJ \& Blackburn, TM (2000) Pattern \& Process in Macroecology. Blackwell Science, Oxford.

Gotelli, NJ (2000) Null model analysis of species co-occurrence patterns. Ecology, 81, 2606-21.

Gotelli, NJ \& Entsminger, GL (2001) Ecosim: Null Models Software for Ecology, Version 6.0. Acquired Intelligence Inc, \& Kesey-Bear http://homepages.together.net/gentsmin/ecosim.htm.

Gotelli, NJ \& Graves, GR (1996) Null Models in Ecology. Smithsonian Institution Press, Washington D.C.

Gotelli, NJ \& Rohde, K (2002) Co-occurrence of ectoparasites of marine fishes: a null model analysis. Ecology Letters, 5, 86-94. 
Guimarães, PR \& Guimarães, P (2006) Improving the analyses of nestedness for large sets of matrices. Environmental Modelling \& Software, 21, 1512-3.

Guimarães, PR, Rico-Gray, V, Furtado dos Reis, S \& Thompson, JN (2006) Asymmetries in specialization in ant-plant mutualistic networks. Proceedings of the Royal Society of London B, 273, 2041-7.

Guimarães, PR, Rico-Gray, V, Oliveira, PS, Izzo, TJ, dos Reis, SF \& Thompson, JN (2007a) Interaction intimacy affects structure \& coevolutionary dynamics in mutualistic networks. Current Biology, $17,1797-803$

Guimarães, PR, Sazima, C, dos Reis, SF \& Sazima, I (2007b) The nested structure of marine cleaning symbiosis: Is it like flowers \& bees? Biology Letters, 3, 51-4.

Handcock, MS (2003) Degreenet: Models for skewed count distributions relevant to networks. Statnet Project, Seattle, WA. Version 1.0, http://www.statnetproject.org.

Handcock, MS, Hunter, DR, Butts, CT, Goodreau, SM \& Morris, M (2003) Statnet: Software Tools for the Statistical Modeling of Network Data. Statnet Project, Seattle, WA. Version 2, http://www.statnetproject.org

Hausdorf, B \& Hennig, C (2007) Null model tests of clustering of species, negative co-occurrence patterns \& nestedness in metacommunities. Oikos, 116, 818-28.

Holland, JN, Okuyama, T \& DeAngelis, DL (2006) Comment on Asymmetric coevolutionary networks facilitate biodiversity maintenance. Science, 313, $1887 \mathrm{~b}$.

Ings TC, Montoya JM, Bascompte, J, Blüthgen, N, Brown, L, Dormann, CF, Edwards, F, Figueroa, D, Jacob, U, Jones, JI, Laurisden, RB, Ledger, ME, Lewis, HM, Olesen, JM, Veen, FJV, Warren, PH \& Woodward, G (2008) Ecological networks - beyond food webs. Journal of Animal Ecology, 78, 253-69.

Jongman, RHG, ter Braak, CJF \& van Tongeren, OFR (1995) Data Analysis in Community \& Landscape Ecology, Cambridge University Press, Cambridge.

Jordano, P (1987) Patterns of mutualistic interactions in pollination \& seed dispersal-connectance, dependence asymmetries, \& coevolution. American Naturalist, 129, 657-77.

Jordano, P, Bascompte, J \& Olesen, JM (2003) Invariant properties in coevolutionary networks of plant-animal interactions. Ecology Letters, 6, 69-81.

Jordano, P, Bascompte, J \& Olesen, JM (2006) In: Waser, NM \& Ollerton, J Eds. The ecological consequences of complex topology \& nested structure in pollination webs. Plant-Pollinator Interactions: From Specialization to Generalization, University of Chicago Press, Chicago, 173-99.

Kato, M, Makutani, T, Inoue, T \& Itino, T (1990) Insect-flower relationship in the primary beech forest of Ashu, Kyoto: an overview of the flowering phenology \& seasonal pattern of insect visits. Biological Laboratory of Kyoto University, 27, 309-75.

Kossinets, G (2006) Effects of missing data in social networks. Social Networks, 28, 247-68.

Krebs, CJ (1989) Ecological Methodology, Harper Collins, New York.

Krebs, CJ (2002) Ecology: The Experimental Analysis of Distribution \& Abundance, 5th ed. Benjamin-Cummings, Zug (CH).

Legendre, P \& Legendre, L (1998) Numerical Ecology, 2nd ed. Elsevier Science.

Lewinsohn, TM, Prado, PI, Jordano, P, Bascompte, J \& Olesen, JM (2006) Structure in plant-animal interaction assemblages. Oikos, 113, 17484.

May, RM (1973) Stability \& Complexity in Model Ecosystems, Princeton University Press, Princeton.

McCann, KS (2000) The diversity-stability debate. Nature, 405, 228-33.

McCune, B, Grace, JB \& Urban, DL (2002) Analysis of Ecological Communities. MjM Software.

McGarigal, K, Cushman, S \& Stafford, S (2000) Multivariate Statistics for Wildlife \& Ecology Research, Springer, New York.

Memmott, J, Waser, NM \& Price, MV (2004) Tolerance of pollination networks to species extinctions. Proceedings of the Royal Society, 271, 2605-11.

Milo, R, Shen-Orr, S, Itzkovitz, S, Kashtan, N, Chklovskii, D \& Alon, U (2002) Network motifs: simple building blocks of complex networks. Science, 298, 824-7.

Montoya, JM, Pimm, SL \& Solé, RV (2006) Ecological networks \& their fragility. Nature, 442, 259-64

Moore, J \& Swihart, R (2007) Toward ecologically explicit null models of nestedness. Oecologia, 152, 763.
Moore, JE \& Swihart, RK (2007) Toward ecologically explicit null models of nestedness. Oecologia, 152, 763-77.

Morris, RJ, Lewis, OT \& Godfray, HCJ (2004) Experimental evidence for apparent competition in a tropical forest food web. Nature, 428 , 310-3.

Motten, AF (1982) Pollination Ecology of the Spring Wildflower Community in the Deciduous Forests of Piedmont North Carolina. $\mathrm{PhD}$ thesis, Duke University, Duhram, North Carolina, USA.

Mouillot, D, Krasnov, BR, Shenbrot, GI \& Poulin, R (2008) Connectance \& parasite diet breadth in flea-mammal webs. Oikos, 31, 16-20.

Müller, CB, Adriaanse, ICT, Belshaw, R \& Godfray, HCJ (1999) The structure of an aphid-parasitoid community. Journal of Animal Ecology, 68, 346-70.

Nielsen, A \& Bascompte, J (2007) Ecological networks, nestedness \& sampling effort. Journal of Ecology, 95, 1134-41.

Novak, M \& Wootton, JT (2008) Estimating nonlinear interaction strengths: an observation-based method for species-rich food webs. Ecology, 89, 2083-9.

Oksanen, J, Kindt, R, Legendre, P, O'Hara, B, Simpson, GL, Stevens, MHH \& Wagner, H (2008) Vegan: Community Ecology Package. R package version 1.13-1, URL http://www.r-project.org.

Olesen, JM, Bascompte, J, Dupont, YL \& Jordano, P (2006) The smallest of all worlds: Pollination networks. Journal of Theoretical Biology, 240, 270-6

Olesen, JM, Bascompte, J, Dupont, YL \& Jordano, P (2007) The modularity of pollination networks. Proceedings of the National Academy of Science USA, 104, 19891-6.

Olesen, JM \& Jordano, P (2002) Geographic patterns in plant-pollinator mutualistic networks. Ecology, 83, 2416-24.

Patefield, WM (1981) Algorithm AS159. An efficient method of generating r x c tables with given row \& column totals. Applied Statistics, 30 91-7.

Patterson, BD \& Atmar, W (1986) Nested subsets \& the structure of insular mammalian faunas \& archipelagos. Biological Journal of the Linnean Society, 28, 65-82.

Petanidou, T, Kallimanis, AS, Tzanopoulos, J, Sgardelis, SP \& Pantis, JD (2008) Long-term observation of a pollination network: fluctuation in species \& interactions, relative invariance of network structure \& implications for estimates of specialization. Ecology Letters, 11, 564-75

Petanidou, T \& Potts, SG (2006) In: Waser, NM \& Ollerton, J Eds. Mutual use of resources in Mediterranean plant-pollinator communities: How specialized are pollination webs? Plant-Pollinator Interactions: From Specialization to Generalization, University of Chicago Press, Chicago, 220-44.

Pimm, SL (1982) Food Webs, Chicago University Press, Chicago.

R Development Core Team (2008) R: A Language \& Environment for Statistical Computing. R Foundation for Statistical Computing, Vienna, Austria, URL http://www.R-project.org.

Rezende, EL, Lavebre, JE, Guimarães, PR, Jordano, P \& Bascompte, J (2007) Non-random coextinctions in phylogenetically structured mutualistic networks. Nature, 448, 925-8.

Roberts, A \& Stone, L (1990) Island-sharing by archipelago species. Oecologia, 83, 560-7.

Rodríguez-Gironés, MA \& Santamaría, L (2006) A new algorithm to calculate the nestedness temperature of presence-absence matrices. Journal of Biogeography, 33, 924-35.

Rohde, K (2001) Spatial scaling laws may not apply to most animal species. Oikos, 93, 499-504.

Santamaría, L \& Rodríguez-Gironés, MA (2007) Linkage rules for plantpollinator networks: Trait complementarity or exploitation barriers? PLoS Biology, 5, 354-62.

Schluter, D (1984) A variance test for detecting species associations, with some example applications. Ecology, 65, 998-1005.

Srinivasan, UT, Dunne, JA, Harte, J \& Martinez, ND (2007) Respone of complex food webs to realistic extinction sequences. Ecology, 88 , 671-82.

Stang, M, Klinkhamer, PGL \& van der Meijden, E (2006) Size constraints $\&$ flower abundance determine the number of interactions in a plant-flower visitor web. Oikos, 112, 111-21.

Stang, M, Klinkhamer, PGL \& van der Meijden, E (2007) Asymmetric specialization \& extinction risk in plant-flower visitor webs: a matter of morphology or abundance? Oecologia, 151, 442-53.

Stone, L \& Roberts, A (1992) Competitive exclusion, or species aggregation? An aid in deciding. Oecologia, 91, 419-24. 
Strong, DR, Simberloff, D, Abele, LG \& Thistle, AB (Eds) (1984) Ecological Communities: Conceptual Issues \& the Evidence, Princeton Univ., Press, Princeton.

Sun, LF, Jiang, L, Li, MH \& He, DC (2006) Statistical analysis of gene regulatory networks reconstructed from gene expression data of lung cancer. Physica A: Statistical Mechanics \& Applications, 370, 663-71.

Tylianakis, J (2008) Understanding the web of life: the birds, the bees, \& sex with aliens. PLoS Biology, 6, e47.

Tylianakis, JM, Tscharntke, T \& Lewis, OT (2007) Habitat modification alters the structure of tropical host-parasitoid food webs. Nature, 445, 202-5.

Ulrich, W \& Gotelli, NJ (2007a) Disentangling community patterns of nestedness \& species co-occurrence. Oikos, 116, 2053-61.

Ulrich, W \& Gotelli, NJ (2007b) Null model analysis of species nestedness patterns. Ecology, 88, 1824-31.

Vázquez, DP \& Aizen, MA (2003) Null model analyses of specialization in plant-pollinator interactions. Ecology, 84, 2493-501.

Vázquez, DP \& Aizen, MA (2004) Asymmetric specialization: A pervasive feature of plant-pollinator interactions. Ecology, 85, 1251-7.

Vázquez, DP \& Aizen, MA (2006) In: Waser, NM \& Ollerton, J Eds. Community-wide patterns of specialization in plant-pollinator interactions revealed by null models. Plant-Pollinator Interactions: From Specialization to Generalization, University of Chicago Press, Chicago, 200-19.
Vázquez, DP, Melián, CJ, Williams, NM, Blüthgen, N, Krasnov, BR \& Poulin, R (2007) Species abundance \& asymmetric interaction strength in ecological networks. Oikos, 116, 1120-7.

Vázquez, DP, Morris, WF \& Jordano, P (2005) Interaction frequency as a surrogate for the total effect of animal mutualists on plants. Ecology Letters, 8, 1088-94.

Vázquez, DP \& Simberloff, D (2002) Ecological specialization \& susceptibility to disturbance: Conjectures \& refutations. American Naturalist, 159, 606-23.

Vázquez, DP \& Simberloff, D (2003) Changes in interaction biodiversity induced by an introduced ungulate. Ecology Letters, 6, 1077-83.

Waser, NM (2006) In: Waser NM \& Ollerton J Eds. Specialization \& generalization in plant-pollinator interactions: a historical perspective. Plant-Pollinator Interactions: From Specialization to Generalization, University of Chicago Press, Chicago, 3-18.

Waser, NM, Chittka, L, Price, MV, Williams, NM \& Ollerton, J (1996) Generalization in pollination systems, \& why it matters. Ecology, 77, 1043-60.

Watts, DJ \& Strogatz, S (1998) Collective dynamics of 'small-world' networks. Nature, 393, 440-2.

Weiher, E \& Keddy, P (Eds) (1999) Ecological Assembly Rules Perspectives, Advances, Retreats, Cambridge University Press, Cambridge.

Williams, RJ \& Martinez, ND (2008) Success \& its limits among structural models of complex food webs. Journal of Animal Ecology, 77, 512-9.

(C) Dormann et al.; Licensee Bentham Open.

This is an open access article licensed under the terms of the Creative Commons Attribution Non-Commercial License (http://creativecommons.org/licenses/by$\mathrm{nc} / 3.0 /$ ), which permits unrestricted, non-commercial use, distribution and reproduction in any medium, provided the work is properly cited. 